\title{
LONG-TERM STUDY OF ERYTHROPOIETIN, LOSARTAN, AND COMBINATION IN STREPT- OZOTOCIN-INDUCED DIABETIC RATS
}

\author{
Byy \\ Rehab H. Ashour*, Abd El-Motaal Fouda*, \\ Mohamed Ahdy A. Saad*, Farida Mohamed A. \\ El-Banna*, Fatma Al-Husseiny Moustafa** \\ Trom \\ Department of Clinical Pharmacology*, Depatment of Pathology ${ }^{\star \star}$, \\ Faculty of Medicine, Mansoura University
}

\begin{abstract}
Recombinant human erythropoietin (rHuEPO) has emerged as a new renoprotective agent against various acute kidney injuries. Experience with rHuEPO in chronic kidney injuries is so far limited and conflicting results were obtained. In the present study, we addressed to evaluate the long-term renal effects of rHuEPO in diabetic nephropathy (DN) of rats in relation to novel hypoxia theory and endogenous EPO secretion. We compared rHuEPO to a standard drug, losartan (LSR), and the possibility of add-on therapy was also tested. Thirty-four male SpragueDawley rats were randomly divided into five groups: control-naïve group, untreated diabetic group, EPO-
\end{abstract}

treated diabetic group (150 U/kg, S.C., TIW), LSR-treated diabetic group (5 mg/kg/day), and EPO-LSRtreated diabetic group. Drug treatment was started one week after streptozotocin (STZ) injection and continued for twenty-eight weeks. STZ-treated diabetic rats developed progressive albuminuria, renal dysfunction, and significant glomerular change 28 weeks after induction of diabetes. Chronic administration of rHuEPO alone or in combination with LSR to the STZ-induced diabetic rat did not show beneficial effect on DN evolution, inspite of improving diabetic-renal hypoxia. The best beneficial effect on DN evolution was obtained by LSR sole therapy based on renal function evaluation, albuminu- 
ria, and renal histopathology. Interestingly, administration of LSR either alone or in combination with rHuEPO in STZ-induced diabetic rats significantly abolished increased plasma endogenous EPO. In conclusion, this study has questioned the renoprotective role of low-dose rHuEPO in the setting of DN and confirmed that this low-dose rHuEPO used had adverse effects on blood pressure and increased hematocrit level.

Key words : Diabetic nephropathy, Erythropoietin, Losartan, Rats.

\section{INTRODUCTION}

Diabetic nephropathy (DN) is one of the most serious complications of diabetes and a leading cause of endstage renal disease (ESRD) worldwide. In fact, about $44 \%$ of new ESRD cases have a primary diagnosis of diabetes (U.S. Renal Data System, 2011). The pathogenesis of DN is complex and implies interactions between different metabolic and hemodynamic factors that act concomitantly to activate intracellular second messengers and various growth factors such as the prosclerotic cytokines, transforming growth factor- $\beta$ (TGF $\beta-1)$, connective tissue growth factor (CTGF), and vascular endothelial growth factor (VEGF) (Vinod, 2012). Activation of local renin-angiotensin system (RAS) played a major role in progression of diabetic glomerulosclerosis (Carey \& Siragy, 2003). In addition, the novel hypoxia theory of chronic kidney disease (CKD) including DN and its role in progression of renal injury is well documented (Heyman et al., 2008). Despite the beneficial effects of the current strategies in the treatment of DN, based mainly on adequate glycemic control and blockade of RAS, albuminuria and progression of renal disease are not completely halted. Therefore, it is necessary to explore potential new renoprotective therapy based on the novel hypoxia theory.

Erythropoietin (EPO) is a $30.4 \mathrm{kD}$ acidic glycoprotein hormone that regulates erythropoiesis and primarily synthesized by renal cortical interstitial fibroblasts (Fisher, 2003). EPO has been used clinically both as an erythropoietic stimulating agent in the treatment of anemia associated with various pathologies and as a tissue-protective agent in certain clinical settings, e.g. stroke, multiple sclerosis and acute myocardial infarction (Konstantinopoulos et al., 
2007). Many studies demonstrated that EPO or recombinant human EPO (rHuEPO) protected against injury caused by ischemia/reperfusion in the brain, vessels, heart, and kidney (Sasaki, 2003). However, few studies have demonstrated the protective effects of rHuEPO on chronic renal injury. Clinical experience with EPO in CKD has been conflicting with some trials showing a beneficial effect on renal failure progression; whereas others have failed to demonstrate any effect (Harris et al., 2006).

Moreover, the tissue-protective effect of EPO was linked to the use of low dose than that used for anemia correction. In animal model for type 2 diabetes, long-term treatment with a long-acting erythropoiesisstimulating agent had striking dosage-dependent effects on molecular pathways of diabetic kidney damage with low-dosage therapy fully exposed the tissue-protective potential (Menne et al., 2007). Moreover, in streptozotocin-induced diabetic rats, one month treatment with the low dose rHuEPO attenuated renal injury beyond its hematopoietic effect (Toba et al., 2009). Whether this tissue-protective effect of low-dose
EPO could be maintained for longer periods without adverse effects is not known. Also, the relation between the renal effects of low-dose EPO and renal hypoxia has not been investigated. So, the aim of this work is to evaluate the long-term renal effects of rHuEPO in DN of rats and its relation to renal hypoxia and endogenous EPO secretion. The effect of rHuEPO was compared to standard drug, losartan (LSR), and the effect of EPO and LSR combination was also investigated.

\section{MATERIALS AND METHODS}

Chemicals and drugs :

Streptozotocin (STZ): was obtained from Sigma-Aldrich Chemical Co., St. Louis, MO, USA. Erythropoietin in the form of epoetin alpha (EPREX® syringes) was supplied as a gift from Janssen-Cilag Co., USA. Losartan (dry powder, 98\% activity) was supplied as a gift from Amriya Pharmaceutical Industries, Alexandria, Egypt.

\section{Animals}

Thirty-four male Sprague-Dawley rats (8 weeks old, weighing 160 to $200 \mathrm{~g}$ ) obtained from Medical Experimental Research Center (MERC), Mansoura Faculty of Medicine were

MANSOURA MEDICAL JOURNAL 
used and included 8 control rats and 26 rats made diabetic by single intraperitoneal injection of STZ $(60 \mathrm{mg} /$ $\mathrm{kg})$ dissolved in citrate buffer (10 $\mathrm{mmol} / \mathrm{L}, \mathrm{pH} 4.5)$. At 1 week post-STZ injection, induction of diabetes was confirmed by measuring tail vein blood-glucose level using GlucoDr ${ }^{\mathrm{TM}}$ super sensor glucometer (one touch technology, Allmedicus, Korea). Rats with fasting blood glucose level more than $280 \mathrm{mg} / \mathrm{dl}$ were considered diabetic and were included in the study (Tesch \& Allen, 2007).

Rats were kept on a regular $12 \mathrm{~h}$ dark/light cycle with free access to standard rat chow and tap water ad libitum. The animals were observed for water intake, food intake, changes in body weight, and general health status during the 28 weeks study period. The study design and protocol was revised and approved by Mansoura Medical Research Ethics committee.

\section{Experimental Design and Groups}

Rats were classified into five groups: 1) the control-naïve group (CNT-naïve, $n=8$ ), received citrate buffer only, 2) untreated diabetic group (DM-unt-28W, $\mathrm{n}=8$ ), received single intraperitoneal injection of

Vol. 42, No. 1 \& 2 Jan. \& April, 2013
STZ as previously described (Tesch \& Allen, 2007) and no drug treatment for 28 weeks after induction of diabetes, 3) EPO-treated diabetic group (DM-EPO-28W, $\mathrm{n}=6$ ), received rHuEPO at $150 \mathrm{U} / \mathrm{kg}$, three times per week, S.C. (Toba et al., 2009), 4) LSR-treated diabetic group (DMLSR-28W, $\mathrm{n}=6$ ) received $\mathrm{LSR}$ at 5 mg/kg/day, P.O., dose selected equals to $56 \mathrm{mg}$ human dose according to (Paget \& Barnes, 1964), and 5) EPO-LSR-treated diabetic group (DM-EPO-LSR-28W, $n=6$ ) received combination of EPO and LSR, in the same doses. Drug treatment was started one week after STZ injection and continued for 28 weeks. Because the study duration was 28 weeks and to avoid animal wastage, severely diabetic rats with blood glucose level more than $500 \mathrm{mg} / \mathrm{dl}$ (data obtained from pilot study) were treated daily with low-dose NPH insulin at $4.1 \pm 1.4 \mathrm{IU} / \mathrm{kg}$ body weight subcutaneously to maintain body weight and prevent ketosis without normalizing hyperglycemia (Gross et al., 2004).

Every 4 weeks, rats in each group were weighed and individually housed in metabolic cage (Nalgene; Nalge Company, Rochester, NY, 
USA) to allow $24 \mathrm{~h}$ urine collection that were analyzed for urinary creatinine (Murray, 1987) and albuminuria (Schosinsky et al., 1987). Final blood pressure was measured by indirect tail-cuff plethysmography with a photoelectric sensor (LE5001 pressure meter, PanLab Technology for Bioresearch) in non-anaesthetized rats. At the end of the study, animals were weighed and anesthetized with thiopental at dosage $12 \mathrm{mg} / 100 \mathrm{~g}$ BW (Waynforth \& Flecknell, 1998), laparotomy was done and the left renal vein was exposed, blood samples were collected from the left renal vein by heparinized syringe and analyzed for renal vein oxygen tension, sodium, and potassium levels (Rapid Point 400, Bayer). One $\mathrm{ml}$ of blood collected from the heart was taken in a test tube containing $50 \mu \mathrm{l}$ EDTA for estimation of complete blood count (Sysmex) and reticulocytic count (RC). The other portion of blood samples were centrifuged with EDTA at $3000 \mathrm{rpm}$ for 10 minutes, plasma were separated and stored at $-30^{\circ} \mathrm{C}$ until further biochemical assay.

\section{Blood chemistry}

The following parameters were determined with the use of commer- cially available kits: glucose (Trinder, 1969) (BioMed-Glucose L.S, Eng Chem for lab technology, Hannover, Germany), insulin (Clark \& Hales, 1991) (DRG ${ }^{\circledR}$ Rat Insulin ELISA kit, DRG Diagnostics, Germany), creatinine (Murray, 1987) (Diamond Diagnostics Company, Hannover, Germany), albuminuria (Schosinsky et al., 1987) (ABC Diagnostics kits, New Damietta, Egypt), iron (Garcic, 1979) (ELI Tech, Zone Industrielle, 61500 SEES France), Total iron binding capacity "TIBC" (Schreiber, 2003) (ELI Tech Clinical Systems, Zone Industrielle, 61500 SEES France) with calculation of transferrin saturation "TS" as (iron/TIBC)X100, ferritin ( $\mathrm{Ng}$ et al., 1983) (Diametra, Via Garibaldi, Italy), plasma active-renin concentration (PRacC) (Morganti et al., 1995) (renin ELISA kit, DRG Diagnostics, Germany), and EPO concentration (Jelkmann \& Wolff, 1991) (Quantikine Mouse/Rat EPO Immunoassay ELISA kit, R\&D Systems, USA).

Morphologic analysis of renal tissue

For all groups, the kidneys were perfused in a retrograde fashion through the abdominal aorta using saline $0.9 \%$ and then $10 \%$ neutral buffered formalin for in situ fixation. The kidneys were harvested, 
weighed, cut longitudinally, and send for pathological evaluation in $10 \%$ neutral buffered formalin. The kidney specimens were coded, embedded in paraffin, and sections of $5-\mu \mathrm{m}$ thickness were cut and evaluated using hematoxylin and eosin, periodic acid- Schiff (PAS) reagent, and Masson trichrome stains and examined by light microscopy.

The glomerular changes developed in untreated diabetic control group at 28th week after induction of diabetes included: mesangial matrix expansion, mesangial hypercellularity, and segmental thickening of GBM. The lesions were focal (affecting less than $50 \%$ of all glomeruli per section) in all diabetic groups. These glomerular changes were scored according to their absence or presence as follow: normal (absence of lesions $=$ score 0 ); presence of any one of the above mentioned glomerular lesions (score 1); presence of two glomerular lesions (score 2); presence of the three glomerular lesions (score 3). Also, untreated diabetic control group developed tubulo-interstitial lesions including interstitial inflammation, mild interstitial fibrosis and tubular atrophy. Vascular lesions presented as arteriolar hyalinosis were also mild. Because tubule-interstitial and vascular lesions were mild, they were scored as absent (score 0) or present (score 1).

\section{Statistical Analysis}

All analyses were conducted using SPSS (version 16.0, SPSS, IL, USA). The data were tested for Gaussian distributions by Kolmogorov-Smirnov test. Descriptive statistics were reported as mean \pm standard deviation (SD) for continuous variables or median (min-max), and frequency for categorical variables. Differences in continuous variables were analyzed by one-way analysis of variance (ANOVA) followed by posthoc multiple comparisons (Scheffé test). Categorical variables were analyzed by Kruskal-Wallis $\mathrm{H}$, Mann-Whitney's, and chi-square or Fisher exact tests (when appropriate). Statistical analysis of albuminuria was performed by analysis of variance according to a general linear model for repeated measures. $P$ value $<0.05$ was considered statistically significant at confidence interval $95 \%$.

\section{RESULTS}

General characteristics of experimental groups 
All diabetic groups showed significant hyperglycemia (P1<0.01), insulinopenia $(P 1<0.001)$, and increase of KW/BW ratio (P1<0.05) compared to CNT-naïve group. On the other hand, LSR-treated diabetic group showed significant decrease of KW/BW ratio compared to untreated- (P2 < 0.05) and EPO-treated (P3 $<0.01$ ) diabetic groups (table 1).

Renal functions: plasma creatinine and creatinine clearance

All diabetic groups showed significant increase of plasma creatinine (P1 < 0.001) compared to CNTnaïve group. The LSR -treated diabetic group showed significant decrease of plasma creatinine compared to untreated- $(P 2<0.001)$ and EPO-treated (P3 < 0.001) diabetic groups; but the combination-treated diabetic group showed significant increase of plasma creatinine (P4 < 0.001) compared to LSR-treated diabetic group (table 1). Untreated-, EPO-treated, and combinationtreated diabetic groups showed significant decrease of creatinine clearance compared to CNT-naïve group (P1 < 0.01); while, LSR-treated diabetic group showed significant increase of creatinine clearance compared to untreated- and EPO-treated diabetic groups $(\mathrm{P} 2 \&$ \& $3<0.001)$ and CNT-naïve group ( $\mathrm{P} 1<0.01)$. The combination-treated diabetic group showed significant decrease of creatinine clearance compared to LSR-treated diabetic group (P4 < 0.001) (table 1).

\section{Albuminuria}

All diabetic groups showed progressive significant increase of albuminuria on repeated measures $(P<$ 0.001 ). In addition, albuminuria was significantly increased in all diabetic groups at each time point compared to CNT-naïve group (P1<0.05). Albuminuria of EPO-treated diabetic group was significantly increased at the $12^{\text {th }}$ week till the end of the study compared to untreateddiabetic control group $\left(\mathrm{P}_{2}<0.001\right)$. LSR-treated diabetic group showed significant decrease of albuminuria at the 12th week till the end of the study compared to untreateddiabetic control group (P2 $<0.01$ ) and EPO-treated diabetic group (P3 $<0.001$ ). At the 12th week till the end of the study, combinationtreated diabetic group showed significant decrease of albuminuria compared to untreated-diabetic control group (P2 < 0.01) and EPO-treated diabetic group ( $\mathrm{P} 3<0.001$ ); but, sig- 
52

nificant increase of albuminuria compared to LSR-treated diabetic group (P4<0.01) (figure 1).

\section{Mean Blood Pressure}

Untreated-diabetic control group showed significant decrease of final Mean Blood Pressure (MBP) compared to control-naïve group $(\mathrm{P} 1<$ 0.001 ). However, final MBP of EPOtreated diabetic group was significantly increased compared to control-naïve and untreated-diabetic control (P1 \& P2 < 0.001) groups. Final MBP of LSR-treated diabetic group was significantly decreased compared to control-naïve, untreated-diabetic control, and EPO-treated diabetic groups (P1, $\mathrm{P} 2$, \& $\mathrm{P} 3<$ 0.001). Also, final MBP of combination-treated diabetic group showed significant decrease compared to EPO-treated diabetic group (P3 < 0.001 ) (table 1).

Plasma active-renin concentration, renal vein oxygen tension, and electrolytes

Untreated-diabetic control group showed significant decrease of final $\mathrm{PR}_{\mathrm{ac}} \mathrm{C}\left(\mathrm{P}_{1}<0.001\right)$ compared to CNT-naïve group. However, EPO, LSR, and combination-treated diabetic groups showed significant in- crease of final $\mathrm{PR}_{\mathrm{ac}} \mathrm{C}$ compared to CNT-naïve group and untreateddiabetic control group $\left(\mathrm{P}_{1} \& \mathrm{P}_{2}<\right.$ 0.01). LSR and combination-treated diabetic groups showed significant decrease of final PRacC compared to EPO-treated diabetic group $\left(\mathrm{P}_{3}<\right.$ 0.05) (table 1).

Untreated diabetic control group, EPO, and LSR-treated diabetic groups showed significant decrease of renal vein oxygen tension compared to CNT-naïve group $\left(\mathrm{P}_{1}<\right.$ 0.01). While, EPO and LSR-treated diabetic groups showed significant increase of renal vein oxygen tension compared to untreated-diabetic control group $\left(P_{2}<0.05\right)$. Combination-treated diabetic group showed significant increase of renal vein oxygen tension compared to untreateddiabetic control group $\left(P_{2}<0.001\right)$, EPO-treated diabetic group $\left(\mathrm{P}_{3}<\right.$ 0.01), and LSR-treated diabetic group $\left(P_{4}<0.01\right)$ (table 1$)$.

Untreated diabetic control and EPO-treated diabetic groups showed significant decrease of renal vein sodium level compared to CNT-naïve group $\left(P_{1}<0.01\right)$. LSR and combination-treated diabetic groups showed significant increase of renal 
vein sodium level compared to EPO- betic group showed significant intreated diabetic group $\left(P_{3}<0.01\right)$ (table 1). Untreated diabetic control group, EPO, and combinationtreated diabetic groups showed significant increase of renal vein potassium level compared to CNT-naïve group $\left(P_{1}<0.05\right)$. LSR-treated diabetic group showed significant decrease of renal vein potassium level compared to untreated- and EPOtreated diabetic groups $\left(P_{2}<0.01\right.$, $\left.P_{3}<0.05\right)$. Combination-treated diabetic group showed significant decrease of renal vein potassium level compared to untreated-diabetic control group $\left(\mathrm{P}_{2}<0.05\right)$; but significant increase compared to LSR-treated diabetic group $\left(P_{4}<0.01\right)$ (table 1$)$.

Hematological parameters, EPO concentration, and Iron status

LSR-treated diabetic group showed significant decrease of RBCs count compared to untreateddiabetic control group $\left(\mathrm{P}_{2}<0.01\right)$. Untreated diabetic control group showed significant decrease of $\mathrm{RC}$ compared to CNT-naïve group ( $\mathrm{P} 1<$ 0.05) (table 2). Untreated- and EPOtreated diabetic groups showed significant increase of final EPO concentration compared to CNT-naïve group (P1<0.001). EPO-treated diacrease of final EPO concentration compared to untreated-diabetic control group (P2 < 0.01). LSR and combination-treated diabetic groups showed significant decrease of final EPO concentration compared to EPO-treated diabetic group (P3 < 0.01). Combination-treated diabetic group showed significant decrease of final EPO concentration compared to untreated- $(P 2<0.001)$ and LSRtreated diabetic groups $(\mathrm{P} 4<0.05)$ (table 2).

Transferrin saturation (TS) and plasma ferritin was significantly decreased in EPO and combinationtreated diabetic groups $(\mathrm{P} 1<0.05)$ compared to CNT-naïve group. EPO-treated diabetic group showed significant decrease of TS compared to untreated diabetic group (P2 < 0.05). LSR and combination-treated diabetic groups showed significant increase of TS compared to EPOtreated diabetic group (P3 < 0.05). Plasma ferritin was significantly decreased in untreated-diabetic groups (P1 < 0.01) compared to CNT- naïve group (table 2).

Renal histopathology

All diabetic groups showed signifi-

MANSOURA MEDICAL JOURNAL 
cant increase of glomerular changes score 28 weeks after induction of diabetes (K-W Chi-square $=42.54, \mathrm{P} \leq$ 0.001 ) (table $3 ; P_{1}<0.01$ ) compared to CNT-naïve group (Figure 2). The LSR-treated diabetic group was the only group that showed significant decreased of glomerular changes score (table $3 ; \mathrm{P}_{2}<0.01$ ) compared to untreated diabetic control group. No significant interstitial fibrosis (Fisher's exact tests, $P_{1}=0.200$ ), inflammation (Fisher's exact tests, P1 $=0.200$ ), or arteriolar hyalinosis (Fisher's exact tests, $P_{1}=0.467$ ) were present in untreated-diabetic control group (table 3, figure 3 ).

EPO-treated diabetic group showed significant increase of glomerular changes score (Figure 4) (table 3; P1 < 0.001), IFTA score (Fisher's exact tests, $P_{1}=0.015$ ), and interstitial inflammation score (Figure 5) (Fisher's exact tests, $P_{1} \leq$ 0.001) compared to CNT-naïve group. In addition, EPO-treated diabetic group showed significant increase of interstitial inflammation score (Fisher's exact tests, $\mathrm{P}_{2}=$ 0.031) compared to untreated diabetic control group and picture of acute tubular injury and degeneration (Figure 6) that is not included in
DN pathological classification or scoring system. LSR-treated diabetic group showed significant decrease of IFTA score (Chi-square test = $\left.6.00, P_{3}=0.014\right)$ and interstitial inflammation score (Chi-square test = $6.00, \mathrm{P}_{3}=0.014$ ) compared to EPOtreated diabetic group (table 3 ).

Combination-treated diabetic group showed significant increase of glomerular changes score (Figure 7) (table 3; $P_{1}<0.01$ ), IFTA score (figure 8) (Fisher's exact tests, $P_{1} \leq$ 0.001 ), interstitial inflammation score (Fisher's exact tests, $\left.P_{1}=0.015\right)$, and arteriolar hyalinosis score (Figure 9) (Fisher's exact tests, $P_{1}=$ 0.003) compared to CNT-naïve group. Moreover, combinationtreated diabetic group showed significant increase of glomerular changes score (table $3 ; \mathrm{P}_{4}<0.05$ ) compared to LSR-treated diabetic group and significant increase of IFTA score (Fisher's exact tests, $P_{2}=0.031$ ) compared to untreated-diabetic control group and LSR-treated diabetic groups (Fisher's exact tests, P4 = 0.002). Also, arteriolar hyalinosis score was significantly increased compared to EPO-treated diabetic groups (Fisher's exact tests, P3 = 0.015) (table 3). 


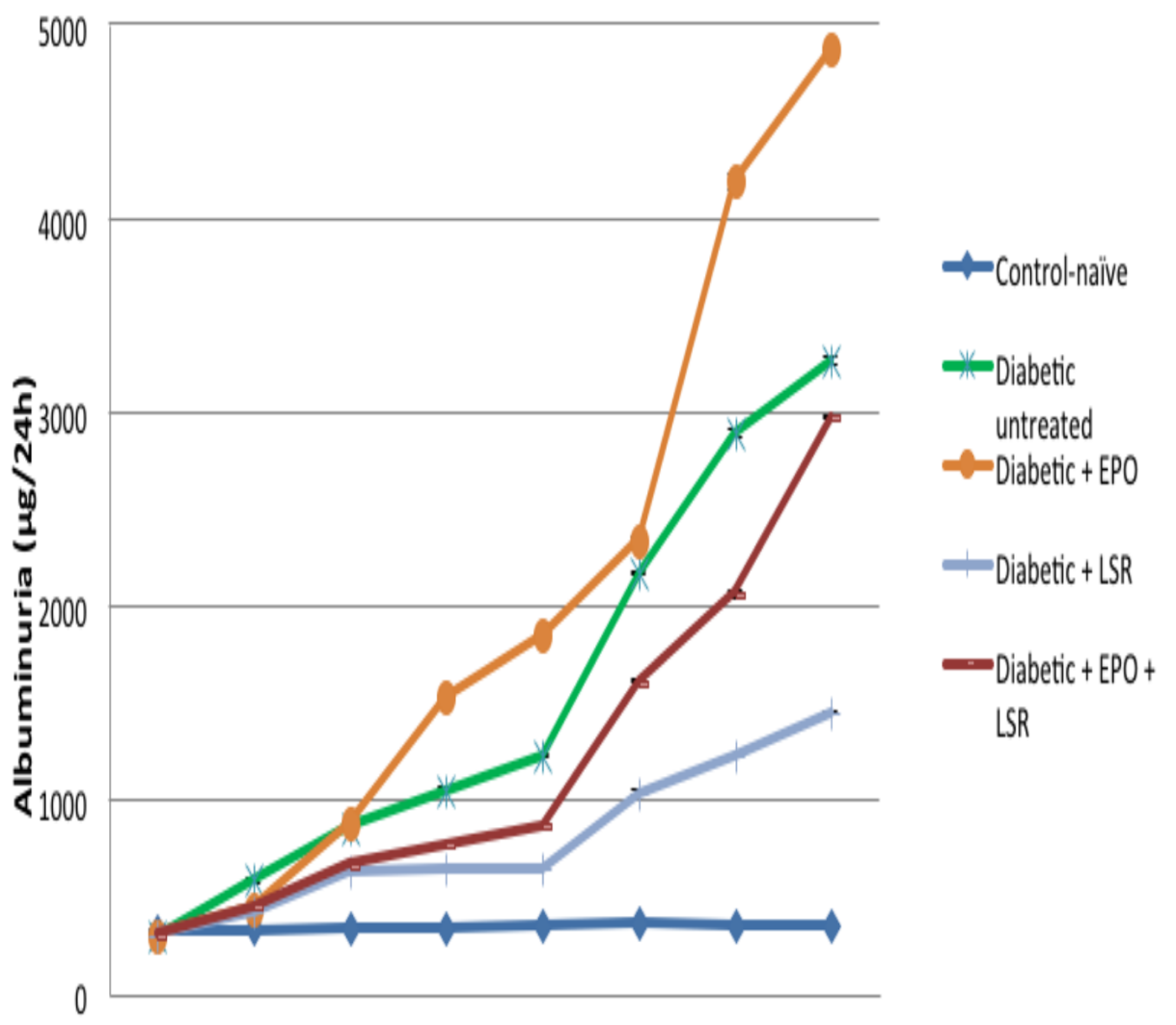

Basal 4 Weeks 8 Weeks 12 Weeks 16 weeks 20 weeks 24 Weeks 28 Weeks

Duration in Weeks

Figure 1: Effect of Erythropoietin (150 U/kg, S.C., TIW), Losartan (5 mg/kg/day, P.O.), or combination on Albuminuria of Control and Diabetic rats. 


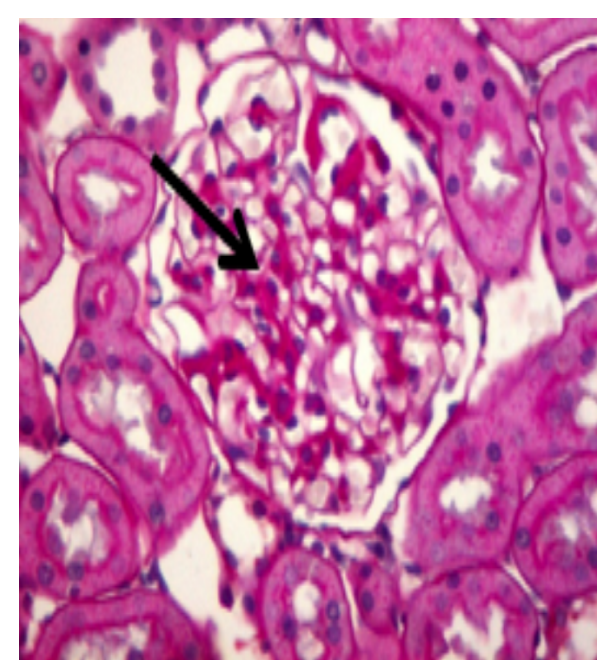

Figure 2: Axial mesangial expansion of 28 weeks untreated diabetic control group (arrow, PAS stain, X400).

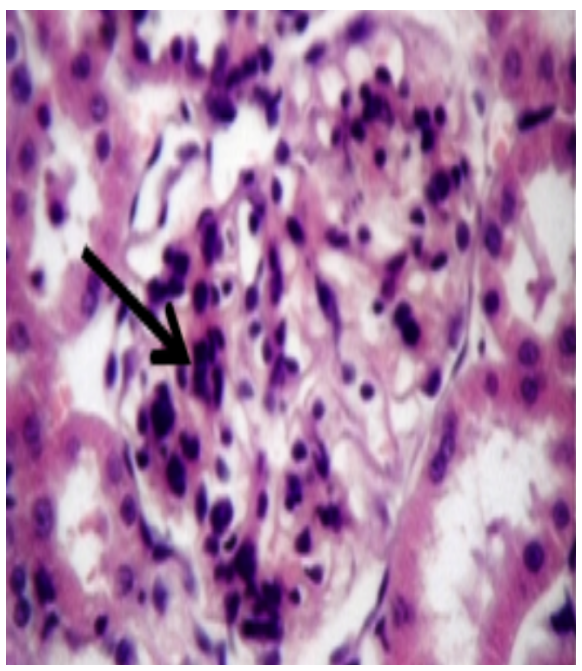

Figure 4: Mesangial hypercellularity of EPOtreated diabetic group (arrow,
PAS stain, X400)

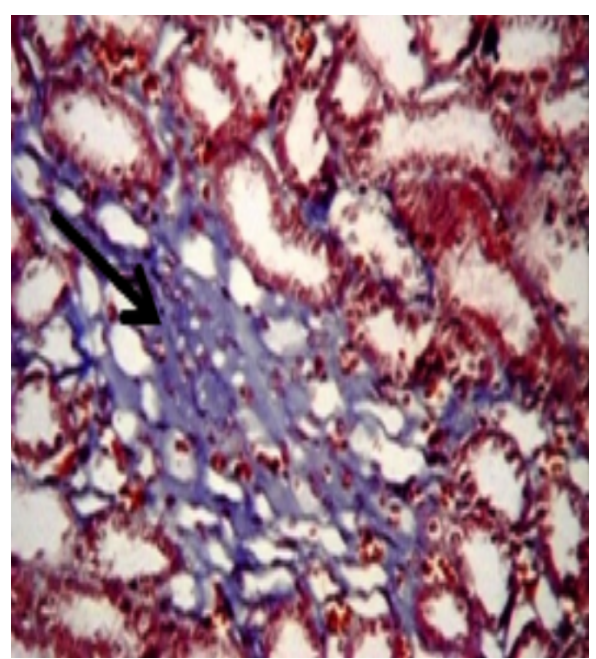

Figure 3: IFTA of 28 weeks untreateddiabetic control group (arrow, Masson Trichrome stain, X250).

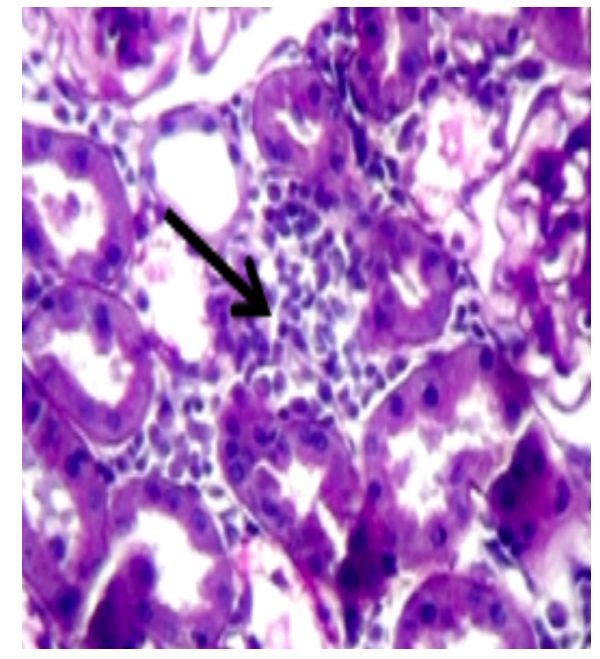

Figure 5: Interstitial inflammatory infiltrate of EPO-treated diabetic group (arrow, PAS stain, X250)

Vol. 42, No. 1 \& 2 Jan. \& April, 2013 


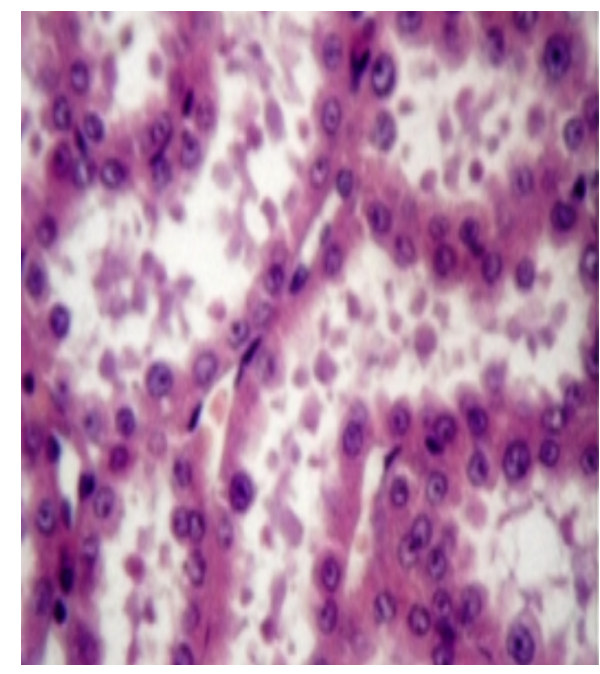

Figure 6: Tubular injury with sheded apical tubular epithelium of EPO-treated diabetic group (Hx\& $E$ stain, $\mathrm{X} 400$ )

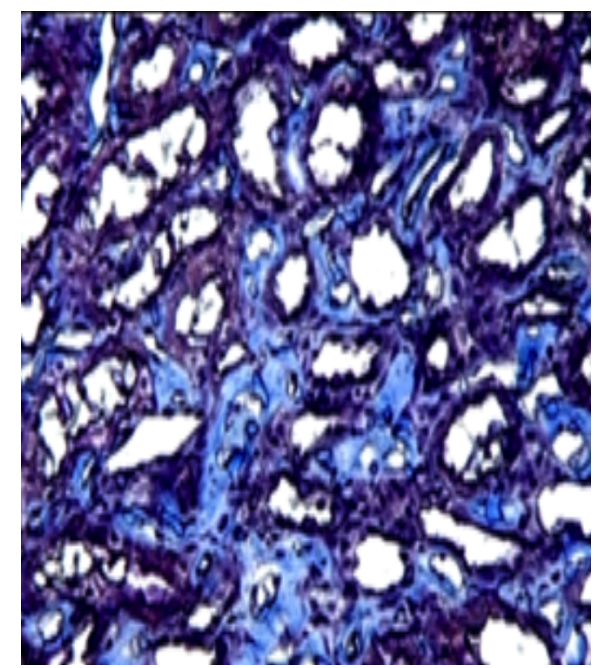

Figure 8: Focal interstitial fibrosis of Combination-treated diabetic group (Masson trichrome stain, X250)

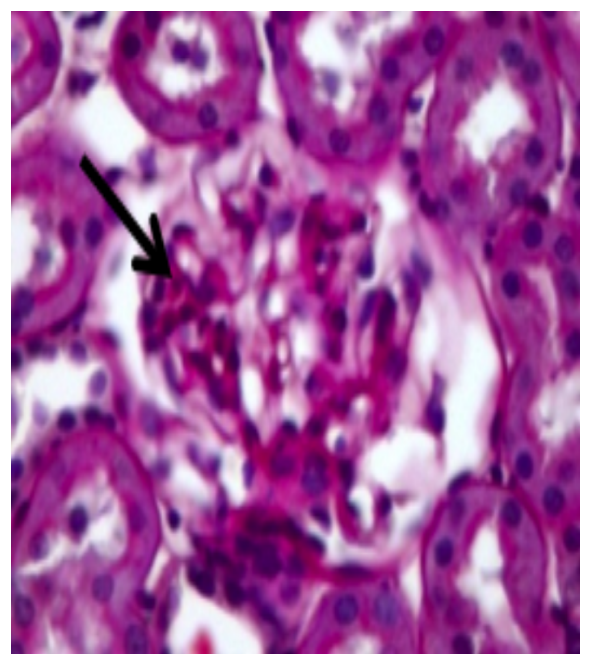

Figure 7: Mesangial expansion with hypercellularity of Combination-treated diabetic group (arrow, PAS stain, $\mathrm{X} 400$ )

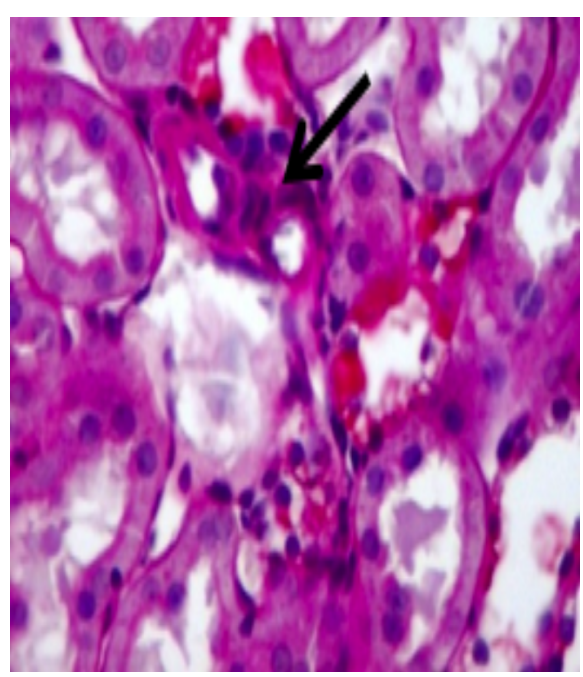

Figure 9: Hyalinized arterioles of Combination-treated diabetic group (arrow, PAS stain, X400)

MANSOURA MEDICAL JOURNAL 
Table 1: General characteristics, Renal functions, Mean Blood Pressure, Plasma active-renin concentration, Renal Vein Oxygen tension, Sodium, and Potassium levels of Different Experimental Groups Followed for 28 Weeks (Mean \pm SD)

\begin{tabular}{|c|c|c|c|c|c|}
\hline & $\begin{array}{c}\text { CNT-naïve } \\
(\mathbf{n}=\mathbf{8})\end{array}$ & $\begin{array}{c}\text { DM-unt- } \\
28 W(n=8)\end{array}$ & $\begin{array}{l}\text { DM-EPO- } \\
28 \mathrm{~W}(\mathrm{n}=6) \\
(150 \mathrm{U} / \mathrm{kg}, \\
\text { S.C., TIW) }\end{array}$ & $\begin{array}{c}\text { DM-LSR- } \\
28 \text { W }(n=6) \\
(5 \\
\text { mg/kg/day, } \\
\text { P.O. })\end{array}$ & 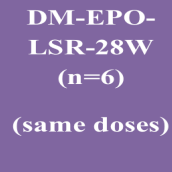 \\
\hline $\begin{array}{l}\text { PI Gl } \\
\text { (mg/dl) }\end{array}$ & $148.8 \pm 17.29$ & $\begin{array}{c}556.9 \pm 86.74 \\
*\end{array}$ & $\begin{array}{c}542.5 \pm 105.4 \\
*\end{array}$ & $\begin{array}{c}539.6 \pm 102.9 \\
*\end{array}$ & $\begin{aligned} 544.9 & \pm 96.41 \\
& *\end{aligned}$ \\
\hline $\begin{array}{l}\text { PI INS } \\
\left(\begin{array}{l}\text { g/L) }\end{array}\right.\end{array}$ & $2.63 \pm 0.23$ & $\begin{array}{c}0.18 \pm 0.03 \\
*\end{array}$ & $\begin{array}{c}0.16 \pm 0.04 \\
*\end{array}$ & $\begin{array}{c}0.1 \pm 0.07 \\
*\end{array}$ & $\begin{array}{c}0.1 \pm 0.08 \\
*\end{array}$ \\
\hline $\begin{array}{l}\mathrm{KW} / \mathrm{BW} \\
(\mathrm{mg} / \mathrm{g})\end{array}$ & $5.48 \pm 0.25$ & $\begin{array}{c}10.28 \pm 1.17 \\
*\end{array}$ & $\begin{array}{c}11.03 \pm 1.07 \\
*\end{array}$ & $\begin{array}{c}7.68 \pm 0.9 \\
* \S\end{array}$ & $\begin{array}{c}10.29 \pm 0.14 \\
* s\end{array}$ \\
\hline $\begin{array}{l}\mathbf{P I ~ C r} \\
(\mathbf{m g} / \mathbf{d l})\end{array}$ & $0.43 \pm 0.03$ & $\begin{array}{c}1.04 \pm 0.07 \\
*\end{array}$ & $\begin{array}{c}1.05 \pm 0.07 \\
*\end{array}$ & $\begin{array}{c}0.63 \pm 0.03 \\
* \S\end{array}$ & $\begin{array}{c}0.96 \pm 0.03 \\
* \kappa\end{array}$ \\
\hline
\end{tabular}

\begin{tabular}{lccccc}
\hline $\begin{array}{l}\text { Cr Cl } \\
(\mathbf{m l} / \mathbf{m i n})\end{array}$ & $1.65 \pm 0.04$ & $1.1 \pm 0.07$ & $1.21 \pm 0.13$ & $4.39 \pm 0.5$ & $1.22 \pm 0.09$ \\
& & $*$ & $*$ & $* \S$ & $* \delta$ \\
\hline $\begin{array}{l}\text { MBP, } \\
\text { final } \\
(\mathbf{m m H g})\end{array}$ & $104.2 \pm 1.58$ & $97.5 \pm 1.41$ & $115.6 \pm 1.86$ & $90.8 \pm 0.75$ & $95.2 \pm 3.65$ \\
\hline
\end{tabular}

\begin{tabular}{|c|c|c|c|c|c|}
\hline $\begin{array}{l}\text { PR }{ }_{\mathrm{ac}} \mathrm{C}, \\
\text { final } \\
(\mathbf{p g} / \mathrm{ml})\end{array}$ & $3.3 \pm 0.43$ & $\begin{array}{c}0.9 \pm 0.31 \\
*\end{array}$ & $\begin{array}{c}59.2 \pm 14.9 \\
* \S\end{array}$ & $\begin{array}{c}6.5 \pm 0.97 \\
* \S\end{array}$ & $\begin{array}{c}21.9 \pm 3.2 \\
* \S\end{array}$ \\
\hline $\begin{array}{l}\mathrm{RV} \mathrm{O}_{2} \mathrm{~T} \\
(\mathrm{mmHg})\end{array}$ & $38.5 \pm 2.69$ & $\begin{array}{c}14.9 \pm 2.62 \\
*\end{array}$ & $\begin{array}{c}27.2 \pm 1.77 \\
* \S\end{array}$ & $\begin{array}{c}25.3 \pm 4.01 \\
* \S\end{array}$ & $\begin{array}{c}41.3 \pm 4.72 \\
\S \diamond \delta\end{array}$ \\
\hline $\begin{array}{l}\mathrm{RV} \mathrm{Na}^{+} \\
(\mathbf{m m o l} / \mathbf{L})\end{array}$ & $133.6 \pm 4.34$ & $\begin{array}{c}121.6 \pm 3.88 \\
*\end{array}$ & $\begin{array}{c}118.1 \pm 3.33 \\
*\end{array}$ & $\begin{array}{c}129.2 \pm 3.18 \\
\end{array}$ & $\begin{array}{c}128.7 \pm 3.12 \\
\end{array}$ \\
\hline $\begin{array}{l}\mathbf{R V ~ K} \mathbf{K}^{+} \\
(\mathbf{m m o l} / \mathbf{L})\end{array}$ & $4.27 \pm 0.78$ & $10.2 \pm 2.06$ & $7.57 \pm 1.43$ & $3.97 \pm 0.47$ & $6.01 \pm 0.51$ \\
\hline
\end{tabular}

Abbreviations: PI GI, Plasma Glucose; PI INS, Plasma Insulin; KW/BW, Kidney-to-Body Weight ratio; PI Cr, Plasma Creatinine; $\mathrm{Cr} \mathrm{Cl}$, Creatinine Clearance; MBP, Mean Blood Pressure; $\mathrm{PR}_{\mathrm{ac}} \mathrm{C}$, Plasma activerenin concentration; RV O2T, Renal Vein Oxygen tension; RV Na+, Renal Vein Sodium level; RV K+, Renal Vein Potassium level

- Statistics: one way ANOVA followed by posthoc Scheffé test, $P$ value $<0.05$.

- $\quad P_{1}(*)$ : Significant difference vs. control-naïve group, $P_{2}(\S)$ : Significant difference vs. DM-unt-28W,

- $\quad P_{3}(\downarrow)$ : Significant difference vs. DM-EPO-28W, $P_{4}(\boldsymbol{\delta})$ : Significant difference vs. DM-LSR-28W.

Vol. 42, No. 1 \& 2 Jan. \& April, 2013 
Table 2: Effect of Erythropoietin (150 U/kg, S.C., TIW), Losartan ( $5 \mathrm{mg} / \mathrm{kg} / \mathrm{day}$, P.O.), or combination on Hematological parameters, EPO concentration, and Iron status of Control and Diabetic rats (Mean \pm SD)

\begin{tabular}{|c|c|c|c|c|c|}
\hline & $\begin{array}{c}\text { CNT-naïve } \\
(n=8)\end{array}$ & $\begin{array}{c}\text { DM-unt- } \\
28 \mathrm{~W}(\mathrm{n}=8)\end{array}$ & $\begin{array}{l}\text { DM-EPO- } \\
28 \mathrm{~W}(\mathrm{n}=6) \\
(150 \mathrm{U} / \mathrm{kg} \\
\text { S.C., TIW) }\end{array}$ & $\begin{array}{c}\text { DM-LSR- } \\
28 W(\mathrm{n}=6) \\
(5 \\
\mathrm{mg} / \mathrm{kg} / \mathrm{day} \\
\text { P.O. }\end{array}$ & $\begin{array}{l}\text { DM-EPO- } \\
\text { LSR-28W } \\
\quad(n=6)\end{array}$ \\
\hline $\begin{array}{l}\text { RBCs } \\
(\mathbf{M} / \quad \mathbf{L})\end{array}$ & $8.2 \pm 0.49$ & $8.5 \pm 0.19$ & $8.2 \pm 1.23$ & $\begin{array}{c}7.9 \pm 0.21 \\
\S\end{array}$ & $8.7 \pm 1.9$ \\
\hline $\begin{array}{l}\text { Hemoglobin } \\
\text { (g/dl) }\end{array}$ & $13.8 \pm 0.41$ & $14.4 \pm 0.94$ & $13.4 \pm 2.13$ & $13.3 \pm 0.42$ & $15.6 \pm 1.34$ \\
\hline $\begin{array}{l}\text { Hematocrit } \\
(\%)\end{array}$ & $45.2 \pm 2.73$ & $48.7 \pm 4.03$ & $50.0 \pm 6.75$ & $43.8 \pm 2.39$ & $53.4 \pm 9.63$ \\
\hline RC (\%) & $2.87 \pm 0.83$ & $\begin{array}{c}1.56 \pm 0.41 \\
*\end{array}$ & $2.33 \pm 0.68$ & $2.08 \pm 0.37$ & $2.83 \pm 0.75$ \\
\hline $\begin{array}{l}\text { EPO, Final } \\
(\mathrm{pg} / \mathrm{ml})\end{array}$ & $38.7 \pm 2.81$ & $\begin{array}{c}53.1 \pm 2.66 \\
*\end{array}$ & $\begin{array}{c}800 \pm 163.4 \\
* \S\end{array}$ & $\begin{array}{c}46.3 \pm 3.84 \\
\bullet\end{array}$ & $\begin{array}{c}35.9 \pm 3.12 \\
\S \triangleleft \delta\end{array}$ \\
\hline Iron ( $\mathrm{g} / \mathrm{dl})$ & $26.4 \pm 1.16$ & $25.8 \pm 2.41$ & $24.4 \pm 2.40$ & $25.7 \pm 3.12$ & $25.5 \pm 2.16$ \\
\hline TS (\%) & $42.6 \pm 3.00$ & $38.7 \pm 1.40$ & $\begin{array}{c}29.1 \pm 4.24 \\
* \S\end{array}$ & $\begin{array}{c}41.2 \pm 4.36 \\
\bullet\end{array}$ & $\begin{array}{c}37.8 \pm 0.89 \\
*\end{array}$ \\
\hline $\begin{array}{l}\text { Ferritin } \\
(\mathrm{ng} / \mathrm{ml})\end{array}$ & $45.7 \pm 4.04$ & $\begin{array}{c}35.8 \pm 2.67 \\
*\end{array}$ & $\begin{array}{c}37.5 \pm 2.27 \\
*\end{array}$ & $\begin{array}{c}41.8 \pm 2.36 \\
\S\end{array}$ & $\begin{array}{c}35.5 \pm 3.34 \\
*\end{array}$ \\
\hline
\end{tabular}

Abbreviations: RBCs, Red Blood Cells; RC, Reticulocytic Count; EPO, Erythropoietin conc.; TS, Transferrin saturation

- Statistics: one way ANOVA followed by posthoc Scheffé test, $P$ value $<0.05$

- $\quad P_{l}(*)$ : Significant difference vs. control-naïve group, $P_{2}(\S)$ : Significant difference vs. DM-unt-28W, $P_{3}$

$(\bullet)$ : Significant difference vs. DM-EPO-28W, $P_{4}(\boldsymbol{\delta})$ : Significant difference vs. DM-LSR-28W.

MANSOURA MEDICAL JOURNAL 
Table 3: Histopathological scores of Control and Diabetic rats

\begin{tabular}{|c|c|c|c|c|c|}
\hline & $\begin{array}{c}\text { CNT-naïve } \\
(\mathrm{n}=\mathbf{8})\end{array}$ & $\begin{array}{l}\text { DM-unt- } \\
28 W(n=8)\end{array}$ & $\begin{array}{l}\text { DM-EPO- } \\
28 W(n=6) \\
(150 \text { U/kg, } \\
\text { S.C., TIW) }\end{array}$ & $\begin{array}{c}\text { DM-LSR- } \\
28 \mathrm{~W}(\mathrm{n}=6) \\
(5 \\
\mathrm{mg} / \mathrm{kg} / \mathrm{day} \\
\text { P.O. }\end{array}$ & $\begin{array}{c}\text { DM-EPO- } \\
\text { LSR-28W } \\
\quad(n=6) \\
\text { (same doses) }\end{array}$ \\
\hline $\begin{array}{l}\text { Glomerular } \\
\text { score } \\
\text { Median } \\
\quad \text { (min-max) }\end{array}$ & $\begin{array}{c}0.00 \\
(0-0)\end{array}$ & $\begin{array}{c}2.00 \\
(1-2) \\
*\end{array}$ & $\begin{array}{c}1.00 \\
(1-2) \\
*\end{array}$ & $\begin{array}{c}1.00 \\
(0-1) \\
* \S\end{array}$ & $\begin{array}{c}2.00 \\
(1-3) \\
* \delta\end{array}$ \\
\hline
\end{tabular}

\section{IFTA score (\%)}

Present $\quad 0.0(n=0 / 8) \quad 37.5(n=3 / 8) \quad 66.7(n=4 / 6) \quad 0.0(n=0 / 6) \quad 100.0(n=6 / 6)$

\begin{tabular}{lccccc} 
& & $*$ & & $* \S \delta$ \\
\hline $\begin{array}{l}\text { Interstitial } \\
\text { Inflammation } \\
(\%)\end{array}$ & & & & & \\
$\quad$ & & & & & \\
$\quad$ Present & $0.0(\mathrm{n}=0 / 8)$ & $37.5(\mathrm{n}=3 / 8)$ & $100.0(\mathrm{n}=6 / 6)$ & $33.3(\mathrm{n}=2 / 6)$ & $66.7(\mathrm{n}=4 / 6)$ \\
& & & $* \S$ & & $*$ \\
\hline
\end{tabular}

\section{Arteriolar}

Hyalinosis (\%)

Present $\quad 0.0(\mathrm{n}=0 / 8) \quad 25.0(\mathrm{n}=2 / 8) \quad 0.0(\mathrm{n}=0 / 6) \quad 0.0(\mathrm{n}=0 / 6) \quad 83.3(\mathrm{n}=5 / 6)$

Abbreviations: IFTA; Interstitial Fibrosis Tubular Atrophy.

- Values are expressed as median (Min - Max) for glomerular score and percentage present lesion for IFTA, Interstitial Inflammation, and Arteriolar Hyalinosis, $P$ value $<0.05$.

- Statistics: Kruskal-Wallis H and Mann-Whitney's tests for glomerular score, chi-square or Fisher's exact tests for IFTA, Interstitial Inflammation, and Arteriolar Hyalinosis scores.

i. $\quad P_{l}(*)$ : Significant difference vs. CNT-naïve group, $P_{2}(\S)$ : Significant difference vs. DM-unt-28W, $P_{3}$ $(\diamond)$ : Significant difference vs. DM-EPO-28W, $P_{4}(\delta)$ : Significant difference vs. DM-LSR-28W.

Vol. 42, No. 1 \& 2 Jan. \& April, 2013 


\section{DISCUSSION}

The present study demonstrated that long-term administration of rHuEPO alone or in combination with LSR to the STZ-induced diabetic rat for 28 weeks did not show beneficial effect on DN evolution compared to LSR sole therapy that had the best beneficial effect. Inspite of improving diabetic-renal hypoxia by rHuEPO alone and combined with LSR, the final renal effects were hazardous in both groups. These results were supported by renal histopathological examination. Strangely, administration of rHuEPO with LSR in STZinduced diabetic rats abolished increased plasma endogenous EPO observed in rHuEPO alone treateddiabetic group. To our knowledge, no available experimental studies have investigated the possible effect of combined administration of EPO and LSR in models of DN.

As shown in the present study, STZ-treated diabetic rats developed progressive albuminuria, renal dysfunction, significant glomerular changes, and mild tubularinterstitial fibrosis 28 weeks after induction of diabetes. These results are in accordance to previous reports demonstrating that single high-dose STZ model of type 1 diabetes developed strong degree of hyperglycemia and albuminuria with moderate degree of renal failure and some histologic changes associated with DN (Tesch \& Allen, 2007 and Rüster \& Wolf, 2010).

Several studies reported that acute pretreatment with rHuEPO preserved renal function against acute ischemia/reperfusion injury (Chatterjee, 2005). Although the possible renoprotective effect of EPO in the setting of chronic kidney injury is of major clinical importance, it has not been settled yet. Previously, the therapeutic effect of EPO in CKD focused on anemia correction with fulldose EPO that might improve renal hypoxia and delay progression to ESRD; however, conflicting results were obtained in different clinical trials (Harris et al., 2006). Moreover, dissociating the tissue-protective effect of EPO from its hematopoietic effect by the use of lower doses was tested in preclinical disease models where chronic treatment with hematologically non-effective dose of darbepoetin, a long-acting rHuEPO analogue, conferred renal vascular and tissue protection in 5/6 nephrectomy remnant kidney model in the rat

MANSOURA MEDICAL JOURNAL 
62 LONG-TERM STUDY OF ERYTHROPOIETIN etc...

(Bahlmann et al., 2004). However, escalating doses of darbepoetin attenuated the protective effects on the remnant kidney tissue and even worsen microvascular renal injury, that is, glomerulosclerosis (Fliser et al., 2006).

In our study, we used rHuEPO in the same dose used by Toba et al. (2009); as they reported that neither blood pressure nor hematocrit (Hct) was affected by the administration of rHuEPO in a dosage of $150 \mathrm{U} / \mathrm{kg}$, TIW, SC for 4 weeks. Contrarily, our results showed that chronic administration of this low dose of rHuEPO for 28 weeks led to elevation of blood pressure and Hct in diabetic and control non-diabetic rats (data not shown).

In agreement of our results that failed to demonstrate beneficial effects of long-term low-dose rHuEPO administration, an earlier experimental study reported that vigorous correction of anemia with rHuEPO (25 $\mathrm{U}, \mathrm{IP}$, twice weekly) in rats with renal ablation is associated with striking acceleration of glomerular injury and greater severity of proteinuria such that values were higher than those in control rats which was explained by aggravation of systemic and glomerular hypertension (Garcia et al., 1988).

In contrast to our results, beneficial effects were reported in genetic experimental models of type 2 diabetes (i.e., the leptin receptor knockout $\mathrm{db} / \mathrm{db}$ mouse), where chronic administration of continuous erythropoietin receptor activator for 14 weeks had beneficial dosedependent effects on molecular pathways of diabetic kidney damage including TGF- $\beta 1$ and nephrin expression in renal tissue. However, only the non-hematologically effective low-dose was clinically renoprotective, whereas high-dose aggravated albuminuria despite clear-cut beneficial molecular effects. Moreover, phlebotomy in high-dose treated mice preserved its tissue protective effect (Menne et al., 2007). Also, one month treatment with rHuEPO (150 $\mathrm{U} / \mathrm{kg}, \mathrm{TIW}, \mathrm{SC}$ ) in STZ-induced diabetic rats significantly attenuated proteinuria, renal fibrosis, and renal elevation in mRNA for TGF- $\beta$, osteopontin and adhesion molecules, with normalization of renal function. These beneficial effects of chronic rHuEPO treatment occurred beyond hematopoiesis (Toba et al., 2009). 
Rehab H. Ashour et al ....

In the present study, LSR pre- non-diabetic kidney diseases that served renal function and structure the magnitude of albuminuria reducthroughout the 28 weeks study period. In agreement of our results, LSR treatment $(2 \mathrm{mg} / \mathrm{kg}$, p.o., 6 weeks) significantly prevented elevated blood pressure, renal dysfunction, renal hypertrophy, and renal structural alteration in STZ-induced diabetic rat model without altering insulin levels (Murali et al., 2003). Also, LSR was found to modulate glomerular sclerosis and decreases the accumulation of collagen type IV by inhibiting TGF- $\beta 1$ and CTGF in rat model of STZ-induced DN followed for 16 weeks (Xu et al., 2008). In addition, LSR exerted structural and functional renoprotection through attenuation of mitochondrial functional impairment in STZ-induced diabetes (De Cavanagh et al., 2008).

In a randomized, double-blind, multicenter, controlled trial, olmesar$\tan (40 \mathrm{mg}$ once daily for a median of 3.2 years) increased the time to onset of microalbuminuria by $23 \%$ in patients with type 2 diabetes, even when blood-pressure control is excellent in olmesartan and placebo groups (Haller et al., 2011). It is well known from studies in diabetic and tion is a strong predictor of subsequent long-term renoprotection (Holtkamp et al., 2011). The benefits of RAS inhibition in diabetes seem to be beyond blood pressure lowering. Pleiotropic renal benefits of ARBs including: inhibition of advanced glycation end products (AGEs) formation, reduction of oxidative stress, improving tubulointerstitial hypoxia, and reduction of interstitial inflammatory cellular infiltrate (Miyata \& van Ypersele de Strihou, 2006). In addition, ARBs induce renoprotection through reduction of glomerular capillary pressure and amelioration of glomerular hyperfiltration (Wolf et al., 2003). Moreover, studies of the glomerular membrane have demonstrated that the size-selective defect for large shunt-like pores in glomerular membrane in DN was partly restored by treatment with losartan 50 $\mathrm{mg} / \mathrm{day}$ for 2 months (Andersen et al., 2000).

In the present study, administration of EPO alone for 28 weeks led to significant increase of final MBP in diabetic rats compared to control-

MANSOURA MEDICAL JOURNAL 
naïve and untreated-diabetic control groups. However, administration of LSR for 28 weeks in diabetic rats significantly lowered final MBP level. Administration of EPO in combination with LSR prevented EPOinduced increase of final MBP that remained significantly lower compared to EPO alone-treated diabetic group. In agreement of our results, one study in rats showed that EPO administration (150 U/kg BW, TIW) for 6 weeks caused an increase in blood pressure in spontaneously hypertensive rats, but not in Wistar Kayato rats, suggesting genetically related susceptibility (Panzacchi et al., 1997). Also, it has been demonstrated that long-term administration of EPO in normal human subjects and patients with CKD provokes arterial hypertension which occurs independently of EPO's hematopoietic effect (Krapf \& Hulter, 2009). Moreover, EPO treatment with prolonged low-dose or high doses for 3 days induced an increase in total peripheral resistance including the cerebrovascular circulation (Rasmussen et al., 2012). The mechanisms had been proposed to involve EPO-induced release of endothelin (Bode-Böger et al., 1996) and inhibition of endothelial nitric oxide synthase (eNOS)- mediated production of nitric oxide (NO) (Scalera et al., 2005). It was reported that hypertension could aggravate glomerular dysfunction in Wistar fatty rats fed on high-salt diet with more generation of reactive oxygen species (ROS) in the kidney (Tomohiro et al., 2007). So, it is possible that elevation of blood pressure in EPO-treated diabetic rats promoted renal injury progression in this study. The present study demonstrated blood pressure-lowering effect of LSR dosage ( $5 \mathrm{mg} / \mathrm{kg}, \mathrm{PO})$ in diabetic rats which might be considered one of the mechanisms for its renoprotection. Moreover, blood pressure-lowering effect of LSR was reported with the use of lower doses (2 $\mathrm{mg} / \mathrm{kg}$, p.o., 6 weeks) in STZinduced diabetic rats (Murali et al., 2003).

In the present study, administration of EPO either alone or in combination with LSR to diabetic rats for 28 weeks produced marked increase of PRacC that was more pronounced when alone. Previously, it was suggested that circulating EPO levels in normal subjects had intrinsic renal effects which were associated with early marked decrease in circulating levels of renin and aldosterone (OI- 
sen et al., 2011). Therefore, we investigated the effects of rHuEPO on PRacC in control non-diabetic rats and we found that rHuEPO significantly decreased PRacC $(0.5 \pm 0.23$ $\mathrm{pg} / \mathrm{ml}, \mathrm{P} 1<0.001)$ in control rats (data not shown). Previous study raised the possibility that plasma renin concentrations decreased secondary to a decrease in absolute renal proximal tubular reabsorption (Olsen et al., 2011) that in turn increase sodium and water delivery to the macula densa producing inverse changes in renin release (Burns et al., 1993). These data were confirmed previously by Lundby et al. (2007) suggesting that excess rHuEPO may downregulate the activity of RAS independent of changes in red blood cell mass, blood volumes and blood pressure thereby maintaining total blood volume within a narrow range with the advantage of increasing $\mathrm{Hb}$ faster than by increasing erythropoiesis (Lundby et al., 2007). Administration of LSR alone for 28 weeks to diabetic rats produced significant increase of PRacC as angiotensin receptor blockers through interruption of RAS negative feedback regulation on Ang II results in increases in plasma Ang II level, which is associated with increase in renin release from juxtaglomerular cells (Paul et al., 2006). At the same time, treatment with ARBs leads to decreased, rather than increased in kidney Ang II levels through prevention of AT1 receptor- mediated stimulation of intrarenal angiotensinogen, leading to decreased intrarenal production of Ang II and subsequent renoprotection (Nishiyama et al., 2004).

Strangely, plasma EPO concentration was significantly increased in untreated and EPO-treated diabetic rats. Administration of LSR in combination with EPO significantly abolished such overshooting of plasma EPO concentration that even was significantly decreased compared to untreated- and LSR-treated diabetic rats. In support of our results, evidence exists to indicate that prolonged administration of rHuEPO results in a suppression of urinary excretion of endogenous EPO (Lasne et al. 2002). Additionally and in agreement with our observations, EPO levels did not differ significantly in patients who were on ARB therapy compared to those who were not (Mojiminiyi et al., 2006). Also, combined Ang II blockade by captopril and LSR was associated with reduction in EPO mRNA expression in 5/ 
6th nephrectomy rat model (Deng et al., 2010).

The relationship between $\mathrm{rHuE}$ PO administration and RAS is of major concern because RAS regulates the production of endogenous EPO (Dunn \& Donnelly, 2007). Ang II administration in humans stimulated EPO synthesis probably through an AT1-dependent pathway (Gossmann et al. 2001) and, contrarily, ACE inhibitors (Pratt et al. 1992) and ARBs decrease the plasma concentration of endogenous EPO (Durmus et al. 2005). Moreover, studies measuring levels of PRA and aldosterone during treatment with rHuEPO in patients with CKD and ESRD mostly revealed conflicting results ranging from unchanged or reduced activity (reviewed in Rosario \& Epstein, 2006). In patients with type 1 diabetes, genetically inherent high activity of the basal RAS was associated with higher levels of EPO compared with patients with a low activity of the basal RAS (Kristensen et al., 2009). So, it is obvious that the effect of rHuEPO administration on RAS varies with different disease state and different medications. Moreover, a geriatric cohort study found a correlation between higher concentration of endogenous EPO and fatal outcome which may reflect a physiological response to an undiagnosed hypoxemia or impaired bone marrow response (den Elzen et al., 2010). In diabetic patients with CKD, elevated endogenous EPO levels were predictive for mortality and were related mainly to markers of inflammation, independent of kidney function (Wagner et al., 2011).

In the present study, we measured renal vein oxygen tension ( $R V$ $\mathrm{O} 2 \mathrm{~T}$ ) as an indicator of renal oxygen consumption and relative hypoxia. We found that administration of either EPO or LSR for 28 weeks to diabetic rats produced significant improvement of $\mathrm{RV} \mathrm{O} 2 \mathrm{~T}$ compared to untreated-diabetic control rats; although values remained significantly lower than control-naïve group. Combined administration of EPO and LSR for 28 weeks to diabetic rats produced more improvement of $\mathrm{RV}$ O2T compared to untreateddiabetic control rats and EPO or LSR alone-treated diabetic groups. AT1receptor blockade acutely increases renal cortical PO2 (Norman et al., 2003) and, if given chronically, losartan attenuated mitochondrial dysfunction in experimental type 1 dia-

Vol. 42, No. 1 \& 2 Jan. \& April, 2013 
betes (De Cavanagh et al., 2008). (HIF) -1 and -2 that regulates numerCollectively, these data provide a ous target genes, including EPO, mechanistic basis for the use of VEGF, hemeoxygenase (HO)-1, ARBs to prevent dysregulation of intrarenal oxygenation in CKD. There is a strong evidence that hypoxia occurs in diabetic kidney mainly due to reduced nitric oxide bioavailability as a result of its quenching by superoxide and increased oxidative stress (Palm, 2006) which leads to reduced efficiency of oxygen utilization necessary for electrolyte transport. Nitric oxide can increase renal oxygenation by increasing oxygen delivery through vasodilatation (Brezis et al., 1991) and reducing renal oxygen consumption by inhibiting tubular sodium reabsorption (Ortiz \& Garvin, 2002) and also by competing with oxygen at the level of cytochrome oxidase within mitochondria (Koivisto et al., 1999).

The chronic hypoxia hypothesis proposed that hypoxic milieu precedes and triggers a fibrotic response leading to renal fibrosis progression (Fine et al., 2000). Renal hypoxia will stimulate hypoxic gene response resulting in early cell survival and hypoxia adaptation. Adequate gene response is mediated through hypoxia-inducible factors NOS, cyclooxygenase-2, and peroxisome proliferator activated receptor $\alpha$-regulated enzyme, which are required in order to normalize tissue O2 tension (Cummins \& Taylor, 2005). However, chronic hypoxia and even increased HIF expression may be pro-fibrotic and induce glomerular injury (Higgins et al., 2007 and Ohtomo et al., 2008). HIF induction has been reported to correlate with tubulointerstitial injury in biopsies from patients with DN (Higgins et al., 2007). Furthermore, it was hypothesized that hypoxia-induced tubulointerstitial injury may induce interstitial fibrosis and rarefaction of peritubular capillaries, and that fibrosis in turn would impair the tubular and interstitial $\mathrm{O} 2$ supply. Taken together, these events would constitute a vicious cycle that aggravates and accelerates renal injury (Palm \& Nordquist, 2011). So the net result is a vicious circle connecting hypoxia, inflammation, and fibrosis.

Regarding renal vein electrolytes, STZ-induced diabetic rats developed significant decrease of $\mathrm{RV} \mathrm{Na}+$ accompanied by significant increase of

MANSOURA MEDICAL JOURNAL 
$\mathrm{RV} \mathrm{K}^{+}$levels compared to control rats. Administration of EPO to diabetic rats for 28 weeks lowered RV $\mathrm{Na}^{+}$and $\mathrm{RV} \mathrm{K}^{+}$than untreateddiabetic control rats, although they did not reach statistical significance. So, it seems that sodium and potassium homeostasis is greatly influenced by the diabetic state and renal function rather than by rHuEPO itself. In agreement with these results, hyperkalemia was observed in STZinduced diabetic rats 60 days after induction of diabetes associated with mild significantly elevated serum creatinine. The author concluded multifactorial causes of hyperkalemia in these diabetic rats including insulinopenia, hyperosmolality, elevated serum creatinine level and hypoaldosteronism with possible contribution of altered distal tubular response to aldosterone (Kim, 1994). Studies in the STZ-induced diabetic rats showed that $\mathrm{Na}^{+}-\mathrm{K}^{+}-$ATPase was affected in several tissues including kidney. The early phase of diabetes (up to week 6 or 7), a stimulatory effect on the enzyme was documented (Fekete et al., 2008). Longer duration of diabetes was followed by a significant functional depression of renal $\mathrm{Na}^{+}-\mathrm{K}^{+}$-ATPase (Vrbjar et al., 2004). Numerous explanation of de- pressed $\mathrm{Na}^{+}-\mathrm{K}^{+}-$ATPase included lack of insulin (Gupta et al., 1996) and C-peptide (Galuska et al., 2011) and altered expression of $\mathrm{Na}^{+}-\mathrm{K}^{+}-$ ATPase molecule with long-lasting diabetes (Tsimaratos et al., 2001).

In the present study, treatment of diabetic rats with LSR produced significant increase of $\mathrm{RV} \mathrm{Na} \mathrm{Na}^{+}$compared to EPO-treated diabetic rats accompanied by significant decrease of $\mathrm{RV} \mathrm{K}^{+}$compared to untreateddiabetic control and EPO-treated diabetic rats. Maintenance of $\mathrm{Na}^{+}-\mathrm{K}^{+}$ balance by LSR administration in STZ-induced diabetic rats copes with renoprotection observed in this group. Combined administration of EPO and LSR for 28 weeks to diabetic rats produced significant increase of $\mathrm{RV} \mathrm{Na}^{+}$compared to EPOtreated diabetic rats accompanied by significant decrease of $\mathrm{RV} \mathrm{K} \mathrm{K}^{+}$compared to untreated-diabetic control rats only; however, it remained significantly higher compared to controlnaïve and LSR-treated diabetic rats. The possible explanation might be related to the decrease of intraglomerular capillary pressure in diabetic kidney by LSR that consequently decreases the pressure natriuresis and helps to preserve plasma sodium 
(Wolf et al., 2003). However, potassium homeostasis is not maintained mostly because of overall renal harmful effects of combination.

We found that rHuEPO $(150 \mathrm{U} / \mathrm{kg}$ BW, TIW, SC) for 28 weeks produced significant increase of RBCs count, $\mathrm{Hb}$ concentration, and $\mathrm{Hct}$ in control non-diabetic rats (data not shown). These results prove that prolonged chronic administration of such low dose of rHuEPO is hematologically effective in control rats. However, this hematopoietic effect failed to be similarly reproduced in diabetic rats treated with EPO for 28 week that showed hematological profile nearly similar to untreated diabetic control group. The possible explanation is resistance to exogenously administered rHuEPO, as well as, endogenously secreted EPO in the hyperglycemic environment owing to chronic low-grade inflammation with its influence on iron homeostasis (EIKhatib, 2009) and increased oxidative stress (Bamgbola, 2011). A number of experimental and clinical studies have demonstrated significant role of various inflammatory molecules including acute phase reactants, inflammatory cytokines, adhesion molecules, and chemokines in the setting of DN (Navarro \& Mora, 2006). These proinflammatory cytokines like interleukin-1 and -6 , interferon- $\gamma$, and tumor necrosis factor down regulate the expression of EPO receptors on erythroid progenitors and disrupt iron recycling by blocking its release from reticuloendothelial cells (Macdougall et al., 2002). Oxidative stress is well documented in diabetes and may participate in EPO resistance by causing lipid peroxidation of red cell membranes (Gallucci et al., 1999). In addition, the pro-oxidative renal tissue down regulates the generation of HIF-1 $\alpha$ protein, thereby reducing EPO synthesis and red cell production (Suliman et al., 2004).

In the present study, inspite untreated and EPO-treated diabetic rats did not develop anemia, the hematopoietic effect of the administered rHuEPO was different in control and diabetic rats. Moreover, Body iron status may provide additional evidence to support the new concept of EPO resistance in this study. EPO-treated diabetic rats showed significant decrease of TS with comparable plasma ferritin compared to untreated-diabetic control rats denoting the development of

MANSOURA MEDICAL JOURNAL 
functional iron deficiency anemia. Combination-treated diabetic rats showed also significant increase of TS compared to EPO-treated diabetic rats. These results may denote some sort of resistance to rHuEPO therapy in hyperglycemic environment. The influence of ACE inhibitors/ ARBs on EPO resistance is controversial. A range of studies have either suggested an etiological role or dismissed it. A prototype study showed there is a higher EPO requirement in dialysis patients who were treated for hypertension with either ACE inhibitors or ARBs compared with those placed on calcium channel blockers (Nakamoto et al., 2004). Polymorphism of insertion/ deletion ACE gene was suggested to have favorable response to ESA in dialysis subjects (Sharples et al., 2006). Furthermore, ACE inhibitors increase plasma level of $\mathrm{N}$-acetylseryl-aspartyllysyl- proline (AcSDKP), a naturally occurring inhibitor of erythropoiesis (Le Meur et al., 2001). In addition, ARBs inhibit erythroid cellular proliferation by blocking Ang II binding to $A T_{1}$ surface receptor (Mohanram et al., 2008).

Our results also highlight a new Vol. 42, No. 1 \& 2 Jan. \& April, 2013 concept in defining EPO resistance that may occur in certain disease states without connecting its definition to the presence of anemia or failure to reach target $\mathrm{Hb}$ inspite of continued rHuEPO therapy. In general, resistance to EPO is presumed in adult subjects if $\mathrm{Hb}$ mass is $<11 \mathrm{~g} / \mathrm{dl}$ (over a 4-6-month period) despite a weekly dose of EPO in excess of $500 \mathrm{IU} / \mathrm{kg}$ or 30, $000 \mathrm{IU} /$ week (Bamgbola, 2011).

The condition known as functional iron deficiency or relative iron deficiency is unique to patients who are being treated with erythropoiesis stimulating agents like rHuEPO because their increased rate of RBCs synthesis overcomes the ability of transferrin-bound circulating iron to provide adequate substrate for $\mathrm{Hb}$ synthesis resulting in problem of iron utilization with low TS while ferritin level remains normal or elevated (Wish, 2006). The defective release of iron from reticuloendothelial cells could be another responsible factor in significant number of patients leading to ineffective EPO therapy. This reticuloendothelial blockade usually occurs in the setting of acute or chronic inflammation and often correlates with a high C-reactive pro- 
tein level and/or a high erythrocyte sedimentation rate (Wish, 2006). Hepcidin is a peptide that is produced by the liver preventing additional exogenous iron absorption and also inhibits the release of iron from the Reticuloendothelial (RE) system to circulating transferrin. Hepcidin activity is increased in the setting of inflammation/infection, primarily through the release of IL-6 by Kupffer cells in the liver (Ganz, 2003). This explains the phenomenon of RE blockade in which storage iron is not released to circulating transferrin, resulting in a normal or high serum ferritin, low TS level, and reduction in circulating iron (El-Khatib, 2009).

In the present study, LSR administration for 28 weeks to diabetic rats led to significant decrease of RBCs number only, although $\mathrm{Hb}$ conc. and $\mathrm{Hct}$ were non-significantly decreased, compared to untreateddiabetic control rats indicating direct effect on erythropoiesis. Combined administration of EPO and LSR to diabetic rats produced better hematological profile than administration of either drug alone, although it is not statistically significant. Regarding iron status, LSR-treated diabetic rats showed plasma ferritin comparable to control-naïve group. So, iron ho- meostasis is not affected by LSR administration. In agreement of our results, ACEls and ARBs may reduce $\mathrm{Hb}$ concentration in patients with hypertension or CKD. The lowering of $\mathrm{Hb}$ observed during treatment with ACEls and ARBs is usually reversible after treatment discontinuation. The underlying mechanisms are at least in part related to blockade of erythropoietic effects of Ang II on red cell precursors and improved renal blood flow secondary to renal efferent vasodilation, which improves oxygenation (Marathias et al., 2004). A post hoc analysis of the RENAAL Study with an average follow-up of 3.4 years indicated that losartanassociated decline in $\mathrm{Hb}$ occurred in patients with type 2 diabetes and nephropathy; however, beneficial effects of losartan treatment in this patient population on ESRD and proteinuria persisted in the presence of $\mathrm{Hb}$ decrease (Mohanram et al., 2008).

In conclusion, the present study stated that LSR is a gold standard drug in management of DN where it delayed the progression of albuminuria and stabilized renal function. In addition, LSR improved diabetic renal hypoxia and prevented hypoxiainduced EPO secretion. Contrarily to

MANSOURA MEDICAL JOURNAL 
the expectations, administration of rHuEPO for 28 weeks led to marked albuminuria progression with profound increase of PRacC and plasma EPO concentration, together with picture of acute tubular injury on renal histopathology examination. Clearly, the combination therapy points to abolishment of the beneficial effect of LSR in experimental DN by the addition of EPO. This may denote that prolonged administration of rHuEPO, even in low dose, has intrinsic harmful renal effect mitigating the effect of a gold standard drug like LSR. This assumption needs confirmation at the molecular level in future experimental studies.

Acknowledgements: Great thanks to Prof. Dr. Manal Fouda, Professor of Clinical Pathology, Hematology Unit, Mansoura Faculty of Medicine, for her kind cooperation. We are grateful to all staff at Medical Experimental Research Center (MERC). This work was supported by Janssen-Cilag Co., USA and Amriya Pharmaceutical Industries, Egypt.

\section{REFERENCES}

1) Andersen $S$, Blouch $K$, Bialek J, Deckert M, Parving HH, and Myers BD (2000) :
Glomerular permselectivity in early stages of overt diabetic nephropathy. Kidney Int 58(5): 2129-2137.

2) Bahlmann FH, Song $R$, Boehm $S M$, Mengel M, von Wasielewski R, Lindschau C, Kirsch T, de Groot K, Laudeley R, Niemczyk E, Güler F, Menne J, Haller H, Fliser D (2004) : Lowdose therapy with the longacting erythropoietin analogue darbepoetin alpha persistently activates endothelial Akt and attenuates progressive organ failure. Circulation 110 : 1006 1012.

3) Bamgbola OF (2011) : Pattern of resistance to erythropoietin-stimulating agents in chronic kidney disease. Kidney International 80: 464-474.

4) Bode-Böger SM, Böger $\mathbf{R H}$, Kuhn M, Radermacher J, and Frölich JC (1996) : Recombinant human erythropoietin enhances vasoconstrictor tone via endothelin-1 and constrictor 
prostanoids. Kidney Int 50: 1255-1261.

5) Brezis M, Heyman SN, Dinour D, Epstein FH, and Rosen $S$ (1991) : Role of nitric oxide in renal medullary oxygenation. Studies in isolated and intact rat kidneys. $J$ Clin Invest 88: 390-395.

6) Burns KD, Homma T, and Harris RC (1993) : The intrarenal renin-angiotensin system. Semin Nephrol 13: 13-30.

7) Carey RM and Siragy HM (2003) : The intrarenal renin-angiotensin system and diabetic nephropathy. TRENDS Endocrinology Metabolism 14(6): 274-281.

8) Chatterjee PK (2005) : Pleiotropic renal actions of erythropoietin. Lancet 365 : 1890-1892.

9) Clark PMS and Hales CN (1991) : Assay of insulin. In: Textbook of Diabetes, Pickup JC and Williams G eds. Blackwell Scientific
Publications. Vol 1: 335347.

10) Cummins EP and Taylor CT (2005) : Hypoxiaresponsive transcription factors. Pflugers Arch 450: 363-371.

11) De Cavanagh EM, Ferder $L$, Toblli JE, Piotrkowski B, Stella I, Fraga CG, and Inserra F (2008) : Renal mitochondrial impairment is attenuated by AT1 blockade in experimental Type I diabetes. Am J Physiol Heart Circ Physiol 294: H456-H465.

12) den Elzen WP, Willems JM, Westendorp RGJ, de Craen AJ, Blauw GJ, Ferrucci L, Assendelft WJ, and Gussekloo J (2010) : Effect of erythropoietin levels on mortality in old age: the Leiden 85-plus Study. CMAJ 182: 1953-1958.

13) Deng A, Arndt MAK, Satriano J, Singh P, Rieg T, Thomson $\mathrm{S}$, Tang $\mathrm{T}$, and Blantz RC (2010) : Renal protecMANSOURA MEDICAL JOURNAL 
tion in chronic kidney disease: hypoxia-inducible factor activation vs. angiotensin II blockade. Am J Physiol Renal Physiol 299: F1365-F1373.

14) Dunn A \& Donnelly $S$ (2007) : The Role of the Kidney in Blood Volume Regulation: The Kidney as a Regulator of the Hematocrit. Am J Med Sci 334(1): 65-71.

15) Durmus A, Dogan E, Erkoc R, Sayarlioglu H, Topal C, and Dilek I (2005) : Effect of valsartan on erythropoietin and hemoglobin levels in stage III-IV chronic kidney disease patients. Int $\mathrm{J}$ Clin Pract 59: 1001-1004.

16) El-Khatib MT (2009) : The Role of Inflammation on Iron and Erythropoietin Resistance. JNRT 2(2): $45-$ 54.

17) Fekete A, Rosta $K$, Wagner $L$, Prokai A, Degrell P, Ruzicska E, Vegh E, Toth M, Ronai K, Rusai K, Somogyi A, Tulassay $T$, Sza- bo AJ, and Ver A (2008) : $\mathrm{Na}+, \mathrm{K}+-\mathrm{ATPase}$ is modulated by angiotensin II in diabetic rat kidney - another reason for diabetic nephropathy? J Physiol 586.22: 5337-5348.

18) Fine LG, Bandyopadhay $D$, and Norman JT (2000) : Is there a common mechanism for the progression of different types of renal diseases other than proteinuria? Towards the unifying theme of chronic hypoxia. Kid Int 75: S22-S26.

19) Fisher JW (2003) : Erythropoietin: Physiology and Pharmacology Update. Exp Biol Med 228: 1-14.

20) Fliser D, Bahlmann $F H$, and Haller H (2006) : EPO: renoprotection beyond anemia correction. Pediatr Nephrol 21: 1785-1789.

21) Gallucci MT, Lubrano $R$, MeIoni C, Morosetti M, Manca di Villahermosa $S$, Scoppi P, Palombo G, Castello MA, and Cascia- 
ni CU (1999) : Red blood cell membrane lipid peroxidation and resistance to erythropoietin therapy in hemodialysis patients. Clin Nephrol 52: 239-245.

22) Galuska D, Pirkmajer S, Barre's R, Ekberg $\mathrm{K}$, Wahren $\mathrm{J}$, and Chibalin AV (2011) : C-Peptide Increases Na,K-ATPase Expression via PKC- and MAP Kinase-Dependent Activation of Transcription Factor ZEB in Human Renal Tubular Cells. PLoS ONE 6(12): e28294: 1-8.

23) Ganz T (2003) : Hepcidin, a key regulator of iron metabolism and mediator of anemia of inflammation. Blood 102: 783-788.

24) Garcia DL, Anderson $S$, Rennket HG, and Brenner BM (1988) : Anemia lessens and its prevention with recombinant human erythropoietin worsens glomerular injury and hypertension in rats with reduced renal mass. Proc Natl Acad Sci
USA 85: 6142-6146.

25) Garcic A (1979) : A highly sensitive, simple determination of serum iron using chromazurol B. Clinica Chimica Acta 94: 115-119.

26) Gossmann J, Burkhardt R, Harder S, Lenz T, Sedlmeyer A, Klinkhardt $U$, Geiger $H$, and Scheuermann E-H (2001) : Angiotensin II infusion increases plasma erythropoietin levels via an angiotensin II type 1 receptor-dependent pathway. Kidney Int 60: 8386.

27) Gross ML, Ritz E, Schoof A, Adamczak $M$, Koch $A$, Tulp O, Parkman A, ElShakmak A, Szabo A, and Amann A (2004) : Comparison of renal morphology in the Streptozotocin and the SHR/N-cp models of diabetes. Laboratory Investigation 84: 452-464.

28) Gupta S, Phipps K, and Ruderman NB (1996) : Differential stimulation of MANSOURA MEDICAL JOURNAL 
76

$\mathrm{Na}+$ pump activity by insulin and nitric oxide in rabbit aorta. Am J Physiol 270(4 Pt 2): H1287-H1293.

29) Haller H, Ito S, Izzo JL, Januszewicz A, Katayama S, Menne J, Mimran A, Rabelink TJ, Ritz E, Ruilope LM, Rump LC, Viberti $G$, for the ROADMAP Trial Investigators (2011) : Olmesartan for the Delay or Prevention of Microalbuminuria in Type 2 Diabetes. N Engl J Med 364:907-917.

30) Harris DCH, Thomas $M$, Johnson DW, Nicholls K, and Gillin A (2006) : Prevention of progression of kidney disease. Nephrology 11 (Suppl 1): S2-S197.

31) Heyman SN, Khamaisi M, Rosen $S$, and Rosenberger C (2008) : Renal Parenchymal Hypoxia, Hypoxia Response and the Progression of Chronic Kidney Disease. Am J Nephrol 28: 998-1006.

32) Higgins DF, Kimura K, Bern- hardt WM, Shrimanker N, Akai $Y$, Hohenstein B, Saito Y, Johnson RS, Kretzler M, Cohen CD, Eckardt K-U, Iwano M, and Haase1 VH (2007) : Hypoxia promotes fibrogenesis in vivo via HIF-1 stimulation of epithelial-tomesenchymal transition. $J$ Clin Invest 117: 3810-3820.

33) Holtkamp FA, De Zeeuw D, De Graeff PA, Laverman GD, Berl T, Remuzzi G, Packham DK, Lewis JB, Parving $\mathrm{HH}$, and Lambers Heerspink HJ (2011) : Albuminuria and blood pressure, independent targets for cardioprotective therapy in patients with diabetes and nephropathy; a post hoc analysis of the combined RENAAL and IDNT trials. Eur Heart $J 32$ (12):1493-1499.

34) Jelkmann $W$ and Wolff $M$ (1991) : Determination of erythropoietin activity in serum. Methods, indications, and interpretation of the data. Dtsch Med Wo- 
chenschr 116(6): 230-234.

35) Kim HJ (1994): Mechanisms of hyperkalemia associated with hyporeninemic hypoaldosteronism in streptozotocin-induced diabetic rats. J Korean Med Sci 9(2): 107115.

36) Koivisto A, Pittner J, Froelich $M$, and Persson AE (1999) : Oxygen-dependent inhibition of respiration in isolated renal tubules by nitric oxide. Kidney Int 55: 23682375.

37) Konstantinopoulos PA, Karamouzis MV, and Papavassiliou AG (2007) : Selective modulation of the erythropoietic and tissueprotective effects of erythropoietin: Time to reach the full therapeutic potential of erythropoietin. Biochimica Biophysica Acta 1776: -9.

38) Krapf R and Hulter HN (2009) : Arterial hypertension induced by erythropoietin and erythropoiesisstimulating agents (ESA).
Clin J Am Soc Nephrol 4: 470-480.

39) Kristensen PL, Høi-Hansen T, Olsen NV, PedersenBjergaard $U$, and Thorsteinsson B (2009) : Erythropoietin during hypoglycaemia in type 1 diabetes: Relation to basal renin-angiotensin system activity and cognitive function. Diab Res Clin Pract 85: 75-84.

40) Lasne F, Martin L, Crepin N, and de Ceaurriz J (2002) : Detection of isoelectric profiles of erythropoietin in urine: differentiation of natural and administered recombinant hormones. Anal Biochem 311: 119-126.

41) Le Meur $Y$, Lorgeot $V$, Comte L, Szelag JC, Aldigier JC, Leroux-Robert C, and Praloran V (2001) : Plasma levels and metabolism of AcSDKP in patients with chronic renal failure: relationship with erythropoietin requirements. Am J Kidney Dis 38: $510-517$.

MANSOURA MEDICAL JOURNAL 
42) Lundby C, Thomsen JJ, Boushel R, Koskolou M, Warberg J, Calbet JAL, and Robach P (2007) : Erythropoietin treatment elevates haemoglobin concentration by increasing red cell volume and depressing plasma volume. J Physiol 578: 309-314.

43) Macdougall IC and Cooper AC (2002) : Erythropoietin resistance: the role of inflammation and proinflammatory cytokines. Nephrol Dial Transplant 17 (Suppl 11): 39-43.

44) Marathias KP, Agroyannis $B$, Mavromoustakos T, Matsoukas $\mathrm{J}$, and Vlahakos DV (2004) : Hematocritlowering Effect Following Inactivation of ReninAngiotensin System with Angiotensin Converting Enzyme Inhibitors and Angiotensin Receptor Blockers. Current Topics Medicinal Chemistry 4(4): 483-486.

45) Menne J, Park JK, Shushakova $\mathbf{N}$, Mengel $M$, Meier $M$, and Fliser D (2007) : The Continuous Erythropoietin Receptor Activator Affects Different Pathways of Diabetic Renal Injury. J Am Soc Nephrol 18: 20462053.

46) Miyata $T$ and van Ypersele de Strihou C (2006) : Renoprotection of angiotensin receptor blockers: beyond blood pressure lowering. Nephrol Dial Transplant 21 (4): 846-849.

47) Mohanram A, Zhang Z, Shahinfar S, Lyle PA, and Toto RD (2008) : The effect of losartan on hemoglobin concentration and renal outcome in diabetic nephropathy of type 2 diabetes. Kidney International 73: 630-636.

48) Mojiminiyi OA, Abdella NA, Zaki MY, EI Gebely SA, Mohamedi HM, and Aldhahi WA (2006) : Prevalence and associations of low plasma erythropoietin in patients with Type 2 diabetes mellitus. Diabet Med 
23(8): 839-844.

49) Morganti A, Pelizzola D, Mantero F, Gazzano G, Opocher G, and Piffanelli A (1995) : Immunoradiometric versus enzymatic renin assay: results of the Italian Multicenter Comparative Study. Italian Multicenter Study for Standardization of Renin Measurement. J Hypertens 13(1): 19-26.

50) Murali B, Umrani $D N$, and Goyal RK (2003) : Effect of chronic treatment with losartan on streptozotocininduced renal dysfunction. Molecular Cellular Biochem 249: 85-90.

51) Murray RL (1987) : Creatinine. In: Pesce AJ and Kaplan LA, eds. Methods in Clinical Chemistry. St. Louis: CV Mosby Co. 10-17.

52) Nakamoto $H$, Kanno $Y$, Okada H, and Suzuki H (2004) : Erythropoietin Resistance in Patients on Continuous Ambulatory Peritoneal Dial- ysis. Advances Peritoneal Dialysis 20: 111- 116.

53) Navarro JF and Mora C (2006) : Diabetes, inflammation, Proinflammatory cytokines, and diabetic nephropathy. The Scientific World J 6, 908-917.

54) $\mathrm{Ng} \mathrm{RH}$, Brown $\mathrm{BA}$, and Valdes R (1983) : Three commercial methods for serum ferritin compared and the high-dose "hook effect" eliminated. Clinical Chemistry 29: 1109-1113.

55) Nishiyama A, Yoshizumi $M$, Rahman $M$, Kobori $H$, Seth DM, Miyatake A, Zhang G-X, Yao L, Hitomi H, Shokoji T, Kiyomoto H, Kimura S, Tamaki T, Kohno $M$, and Abe $Y$ (2004) : Effects of AT1 receptor blockade on renal injury and mitogen-activated protein activity in Dahl saltsensitive rats. Kidney Int 65:972-981.

56) Norman JT, Stidwill $R$, Singer $M$, and Fine LG (2003) : MANSOURA MEDICAL JOURNAL 
80

Angiotensin II blockade augments renal cortical microvascular pO2 indicating a novel, potentially renoprotective action. Nephron Physiol 94: p39-46.

57) Ohtomo S, Nangaku M, Izuhara $\mathbf{Y}$, Takizawa $\mathrm{S}$, Strihou CY, and Miyata $\mathbf{T}$ (2008) : Cobalt ameliorates renal injury in an obese, hypertensive type 2 diabetes rat model. Nephrol Dial Transplant 23:11661172.

58) Olsen NV, AachmannAndersen NJ, Oturai $\mathbf{P}$, Andersen TM, Rasmussen $A B$, Hulston $C$, Holstein-Rathlou N-H, Robach $\mathbf{P}$, and Lundby C (2011) : Recombinant human erythropoietin in humans down-regulates proximal renal tubular reabsorption and causes a fall in glomerular filtration rate. J Physiol 589: 12731281.

59) Ortiz PA and Garvin JL (2002) : Role of nitric oxide in the regulation of nephron transport. Am J Physiol Renal Physiol 282: F777F784.

60) Paget GE and Barnes JM (1964) : Toxicity tests. Chapt. 6, p.135-166. In: Evaluation of drug activities: Pharmacometrics, vol. I. edited by Laurence DR and Bacharach AL, academic press, London and New York.

61) Palm F (2006) : Intrarenal Oxygen in Diabetes and a Possible Link to Diabetic Nephropathy.

Clinical Experimental Pharmacol Physiol 33: 997-1001.

62) Palm $F$ and Nordquist $L$ (2011) : Renal Tubulointerstitial Hypoxia: Cause and Consequence of Kidney Dysfuction. Clin Exp Pharmacol Physiol 38(7): 424430.

63) Panzacchi G, Pieruzzi F, Castoldi G, Busca G, Bolla GB, Buccianti G, Radice F, Fava C, Martini I, Zan- 
chetti A, Golin R, and Stella A (1997) : Effect of erythropoietin administration on blood pressure and urinary albumin excretion in rats. Am J Hypertens 10(7): 772-778.

64) Paul M, Mehr AP, and Kreutz R (2006) : Physiology of local renin-angiotensin systems. Physiol Rev 86:747803.

65) Pratt MC, Lewis-Barned NJ, Walker RJ, Bailey RR, Shand $\mathrm{BI}$, and Livesey $\mathrm{J}$ (1992) : Effect of angiotensin converting enzyme inhibitors on erythropoietin concentrations in healthy volunteers. $\mathrm{Br} \mathrm{J}$ Clin Pharmacol 34: 363-365.

66) Rasmussen P, Kim YS, Krogh-Madsen R, Lundby C, Olsen NV, Secher NH, van Lieshout JJ (2012) : Both acute and prolonged administration of EPO reduce cerebral and systemic vascular conductance in humans. FASEB J 26 (3):1343-8.
67) Rosario $R$ and Epstein $M$ (2006) : Review: Relationship between erythropoietin administration and alterations of renin-angiotensinaldosterone. Journal of Renin-Angiotensin-

Aldosterone System 7, 135-138.

68) Rüster $C$ and Wolf $G$ (2010) : Models of diabetic nephropathy. Drug Discovery Today: Disease Models 7 (1-2): 35-41.

69) Sasaki R (2003) : Pleiotropic Functions of Erythropoietin. Int Med 42: 142-149.

70) Scalera F, Kielstein JT, Martens-Lobenhoffer J, Postel SC, Tager M \& BodeBoger SM (2005) : Erythropoietin increases asymmetric dimethylarginine in endothelial cells: role of dimethylarginine dimethylaminohydrolase. J Am Soc Nephrol 16: 892-898.

71) Schosinsky KH, Verges $M$, Esqulvel $A L$, and Chavarria MA (1987) : Simple MANSOURA MEDICAL JOURNAL 
82

spectrophotometric determination of urinary albumin by dye-binding with use of bromphenol blue. Clin Chem 33(2): 223-226.

72) Schreiber WE (2003) : Iron, porphyrin, and bilirubin metabolism. In: Clinical Chemistry: Theory, Analysis, Correlation, 4th Ed., Kaplan LA, Pesce AJ, and Kazmierczak SC, Mosby Inc, St Louis, USA, 657 and appendix.

73) Sharples EJ, Varagunam M, Sinnott PJ, McCloskey DJ, Raftery MJ, and Yaqoob MM (2006) : The effect of proinflammatory cytokine gene and angiotensin-converting enzyme polymorphisms on erythropoietin requirements in patients on continuous ambulatory peritoneal dialysis. Perit Dial Int 26: 6468.

74) Suliman HB, Ali M, and Piantadosi CA (2004) : Superoxide dismutase-3 promotes full expression of the
EPO response to hypoxia.

Blood 104: 43-50.

75) Tesch GH and Allen TJ (2007) : Rodent models of streptozotocin-induced diabetic nephropathy. Nephrology 12 : 261-266.

76) Toba H, Sawai $N$, Morishita M, Murata S, Yoshida M, Nakashima K, Morita $Y$, Kobara $M$, and Nakata $T$ (2009) : Chronic treatment with recombinant human erythropoietin exerts renoprotective effects beyond hematopoiesis in streptozotocin-induced diabetic rat. Eur J Pharmacol 612: 106-114.

77) Tomohiro T, Kumai T, Sato $T$, Takeba Y, Kobayashi S, and Kimura K (2007) : Hypertension aggravates glomerular dysfunction with oxidative stress in a rat model of diabetic nephropathy. Life Sciences 80: 1364-1372.

78) Trinder $P$ (1969) : Determina- 
tion of glucose in blood using glucose oxidase with an alternative oxygen acceptor. Ann Clin Biochem 6: 24-27.

79) Tsimaratos $M$, Coste TC, Djemli-Shipkolye A, Daniel L, Shipkolye F, Vague P, and Raccah D (2001) : Evidence of timedependent changes in renal medullary $\mathrm{Na}, \mathrm{K}$ ATPase activity and expression in diabetic rats [abstract]. Cell Mol Biol (Noisy-legrand) 47: 239245.

80) U.S. Renal Data System, USRDS (2011) : 2011 annual data report: Atlas of end-stage renal disease in the United States. Bethesda, MD: National Institutes of Health, National Institute of Diabetes and Digestive and Kidney Diseases. From URL: http:// www.usrds.org/2011/view/ default.asp.

81) Vinod PB (2012) : Pathophysiology of diabetic nephrop- athy. Clinical Queries: Nephrology 0102: 121-126.

82) Vrbjar N, Strelková $S$, _tefek M, Kyselová Z, and Gajdosíková A (2004) : Effect of the pyridoindole antioxidant stobadine on sodium handling of renal $\mathrm{Na}, \mathrm{K}$ ATPase in rats with streptozotocin-induced diabetes. Acta Diabetol 41: 172-178.

83) Wagner M, Alam A, Zimmermann J, Rauh K, KoljajaBatzner A, Raff U, Wanner C, and Schramm L (2011) : Endogenous Erythropoietin and the Association with Inflammation and Mortality in Diabetic Chronic Kidney Disease. Clin J Am Soc Nephrol 6: 1573-1579.

84) Waynforth HB and Flecknell PA (1998) : Experimental and surgical techniques in rats. Academic press, London, 2nd Edition, 66-113.

85) Wish JB (2006) : Assessing Iron Status: Beyond Serum Ferritin and Transferrin MANSOURA MEDICAL JOURNAL 
84 LONG-TERM STUDY OF ERYTHROPOIETIN etc...

Saturation. Clin J Am Soc

Physiol 93(1): P3-13.

Nephrol 1: S4-S8.

86) Wolf G, Butzmann $U$, and

87) $\mathrm{Xu}$ JY, Tao LJ, Wang L, Ning Wenzel UO (2003) : The renin-angiotensin system and progression of renal disease: from hemodynamics to cell biology. Nephron WB, and Liu JS (2008) : The effect of losartan on glomerular sclerosis in rats with diabetic nephropathy. $J$ Cent South Univ (Med Sci) 33(9): 836-840. 


\title{
الملحص العربى \\ دراسة طويلة الملدى لإررثروبيوتين، لوسارتان، \\ والاثنين فى الجرذان المصابة بالسكرى \\ المستحث بالإستربتوزوتوسين
}

\begin{abstract}
لتقد برز الإرثروبويتين (rHuEPO) كعقار جديد حامى للكلية ضد مختلف الإصابات الكلوية الحاده. الخبرة مـع الإرثروبويتين في إصـابات الكلى المزمنه حتى الآن محدودة مـ الحصول على نتائج متضـاربة. في هذه الدراسة، تناولنا تقييم الآثار الكلويه طويلة الأجل للإرثروبويتين فى اعتلال الكلى السكرى فى الجرذان

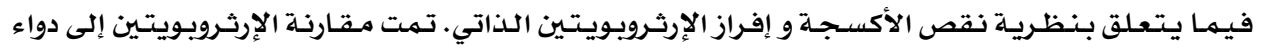
قياسى، لوسـارتان (LSR) و أيضا تم اختبار إمكانية إضافة كلا من العلاجين معا. تم تقسيه أربعة وثثلاثين ذكر من الجـرذان عشوائيـا إلى خمس مسجمـوعات: مـجموعة ضابطة ، مـجمـوعة ضـابطة سكريـة ، مسجموعة

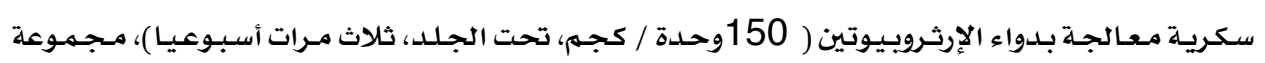

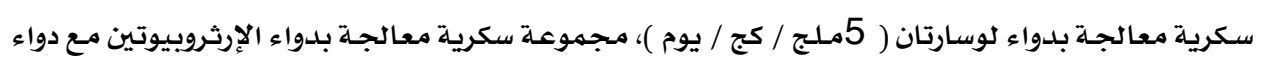

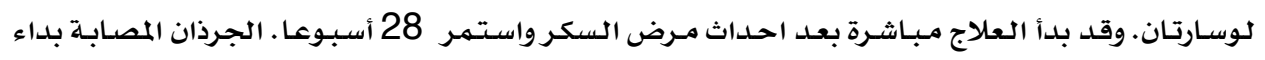
السكري المستحث بـالإستربتوزوتوسين أصسيبت بزيادة الألبومـين تدريـجيا فى البـول، اختالال كلوى، وتغير كبيبي كبير بعد 28 أسبوعا من احداث مرض السكر. الاعطاء المزمن للإرثروبويتين أو بـالاشتراك مـع لوسارتان إلى الجرذان المصابة بالسكري المستحث بالإستريتوزوتوسين لم يظهر تأثير مفيد على تطور اعتلال الكلى السكرى، علي الرغم من تحسين نقص الأكسجة الكلوي السكري. لوسـارتان كعلاج وحيد كان له تأثير مفيد على تطور اعتلال الكلى السكرى معتمـدا على تقييم وظائف الكلى، الألبومين فى

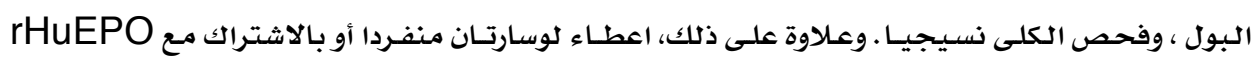

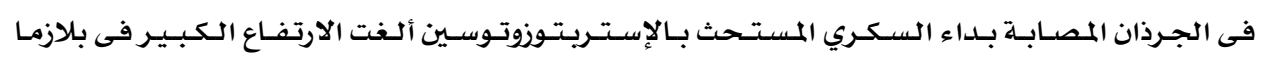
الإرثروبويتـين الذاتى. وفى الختام، هـذه الدراسـة شككت فى الدور الحسامى للكلى للـجرعة المنـخفضدة من كلإرثروبويتين فى اعتلال الكلى السكرى وأثبتت أن هذه الجرعة المنخفضـة من الإرثروبويتين كان لها آثار جانبية على ضغط الدم و أدت الى زيادة مستوى الهيماتوكريت . (Hct).
\end{abstract} MANSOURA MEDICAL JOURNAL 